\title{
Transcriptional Reprogramming and Constitutive PD-L1 Expression in Melanoma Are Associated with Dedifferentiation and Activation of Interferon and Tumour Necrosis Factor Signalling Pathways
}

\author{
Antonio Ahn ${ }^{1}$, Euan J. Rodger ${ }^{1,2}{ }^{(D}$, Jyoti Motwani ${ }^{1}$, Gregory Gimenez ${ }^{1}$, Peter A. Stockwell ${ }^{1}$ (D), \\ Matthew Parry ${ }^{3}\left(\mathbb{D}\right.$, Peter Hersey ${ }^{4}$, Aniruddha Chatterjee ${ }^{1,2, *,+}+\mathbb{D}$ and Michael R. Eccles ${ }^{1,2, *,+}+\mathbb{D}$
}

1 Department of Pathology, Dunedin School of Medicine, University of Otago, 270 Great King Street, Dunedin 9054, New Zealand; antonio.ahn@petermac.org (A.A.); euan.rodger@otago.ac.nz (E.J.R.); mail.jyotim@gmail.com (J.M.); gregory.gimenez@otago.ac.nz (G.G.); peter.stockwell@otago.ac.nz (P.A.S.)

2 Maurice Wilkins Centre for Molecular Biodiscovery, Level 2, 3A Symonds Street, Auckland 1010, New Zealand

3 Department of Mathematics \& Statistics, University of Otago, 710 Cumberland Street, Dunedin 9054, New Zealand; mparry@maths.otago.ac.nz

4 Melanoma Immunology and Oncology Group, The Centenary Institute, University of Sydney, Camperdown, NSW 2050, Australia; peter.hersey@sydney.edu.au

check for updates

Citation: Ahn, A.; Rodger, E.J.; Motwani, J.; Gimenez, G.; Stockwell, P.A.; Parry, M.; Hersey, P.; Chatterjee, A.; Eccles, M.R. Transcriptional Reprogramming and Constitutive PD-L1 Expression in Melanoma Are Associated with Dedifferentiation and Activation of Interferon and Tumour Necrosis Factor Signalling Pathways. Cancers 2021, 13, 4250. https://doi.org/10.3390/cancers 13174250

Academic Editors: Alan Hutson and Song Liu

Received: 15 June 2021

Accepted: 13 August 2021

Published: 24 August 2021

Publisher's Note: MDPI stays neutral with regard to jurisdictional claims in published maps and institutional affiliations.

Copyright: (c) 2021 by the authors. Licensee MDPI, Basel, Switzerland. This article is an open access article distributed under the terms and conditions of the Creative Commons Attribution (CC BY) license (https:// creativecommons.org/licenses/by/ $4.0 /)$.
* Correspondence: aniruddha.chatterjee@otago.ac.nz (A.C.); michael.eccles@otago.ac.nz (M.R.E.); Tel.: +64-3479-7878 (M.R.E.)

+ Joint senior authors.

Simple Summary: Melanoma, an aggressive form of skin cancer, is frequently associated with drug resistance in the advanced stages. For instance, frequently resistance is observed in sequential treatment of melanoma with targeted therapy and immunotherapy. In this research, the authors investigated whether potential transcriptional mechanisms and pathways associated with PD-L1 protein expression could underlie targeted therapy drug resistance in melanoma. The authors found a PD-L1 expression transcriptional pattern underlies resistance to targeted therapy in a subgroup of melanomas. These melanomas were markedly dedifferentiated, as compared to melanomas that were not drug resistant. Understanding changes in transcription and molecular pathways that lead to drug resistance could allow researchers to develop interventions to prevent drug resistance from occurring in melanoma, which could also be relevant to other cancer types.

Abstract: Melanoma is the most aggressive type of skin cancer, with increasing incidence worldwide. Advances in targeted therapy and immunotherapy have improved the survival of melanoma patients experiencing recurrent disease, but unfortunately treatment resistance frequently reduces patient survival. Resistance to targeted therapy is associated with transcriptomic changes and has also been shown to be accompanied by increased expression of programmed death ligand 1 (PD-L1), a potent inhibitor of immune response. Intrinsic upregulation of PD-L1 is associated with genome-wide DNA hypomethylation and widespread alterations in gene expression in melanoma cell lines. However, an in-depth analysis of the transcriptomic landscape of melanoma cells with intrinsically upregulated PD-L1 expression is lacking. To determine the transcriptomic landscape of intrinsically upregulated PD-L1 expression in melanoma, we investigated transcriptomes in melanomas with constitutive versus inducible PD-L1 expression (referred to as PD-L1 $1_{\mathrm{CON}}$ and PD-L1 $1_{\mathrm{IND}}$ ). RNA-Seq analysis was performed on seven PD-L1 $1_{\mathrm{CON}}$ melanoma cell lines and ten melanoma cell lines with low inducible PD-L1 $1_{\text {IND }}$ expression. We observed that PD-L1 $1_{C O N}$ melanoma cells had a reprogrammed transcriptome with a characteristic pattern of dedifferentiated gene expression, together with active interferon (IFN) and tumour necrosis factor (TNF) signalling pathways. Furthermore, we identified key transcription factors that were also differentially expressed in PD-L1 $1_{C O N}$ versus PD-L1 $1_{I N D}$ melanoma cell lines. Overall, our studies describe transcriptomic reprogramming of melanomas with PD-L1 $1_{\text {CON }}$ expression. 
Keywords: melanoma; PD-L1; drug resistance; immunotherapy resistance; dedifferentiation; RNASeq; gene expression; transcriptome; LncRNA

\section{Introduction}

Melanoma is the most deadly form skin cancer, as it frequently presents with highly aggressive features, including high propensity to metastasise and innate drug resistance. Moreover, these features frequently occur at a relatively early stage in the growth of the tumour [1]. Treatment of metastatic melanoma has been revolutionized over the last decade, as greater understanding has emerged of two critical hallmarks of melanoma. Firstly, a large proportion of melanomas (50-65\%) are addicted to MAPK signalling through $B R A F$ or NRAS mutations. In keeping with this, inhibition of the oncogenic BRAF protein has resulted in significant response rates in $B R A F$ mutant melanomas. Secondly, irrespective of mutation status, melanoma is frequently dependent on immune suppression through programmed death 1 (PD1) signalling upon the binding of ligand, either PD-L1 or PDL2 [2]. Anti-PD1 therapy, which inhibits binding of PD-L1 or PD-L2 to the PD1 receptor, reactivates immune responses and has greatly improved melanoma patient survival [3]. Unfortunately, for both of these therapies, resistance inevitably develops, and currently no robust biomarker has been identified that is able to predict patient response. Neither PD1 nor PD-L1/PD-L2 expression accurately predict response, and the basis for intrinsic resistance to anti-PD1 treatment of melanoma is incompletely understood. Nevertheless, PD-L1 is constitutively expressed in some melanomas despite the absence of immune cell infiltration in the tumour [4-6]. Furthermore, PD-L1 has also been shown to be upregulated upon development of resistance to MAPK pathway inhibitors, and it is accompanied by tumour cell-intrinsic transcriptomic reprogramming [4,5]. However, it is currently unclear how intrinsic transcriptomic reprogramming occurs.

As we have previously described [6,7] PD-L1 expression in melanoma can broadly be categorised into mechanisms that are mediated by the presence or absence of tumour infiltrating lymphocytes (TILs). In the presence of TILs, tumour-associated PD-L1 expression is predominantly induced via interferons (IFN) [8-10] and/or cytokines, such as tumour necrosis factor (TNF) [11] secreted from TILs. We refer to melanoma cells with inducible PD-L1 expression as PD-L1 IND. In contrast, PD-L1 expression without TILs is largely mediated cell-intrinsically (or constitutively) via genetic or epigenetic mechanisms, and we refer to these types of melanoma cells as PD-L1 $1_{\mathrm{CON}}[2,12]$.

In this study, we describe transcriptomic features of melanoma cell lines with constitutive high expression of PD-L1 (PD-L1 $1_{C O N}$ ) compared to melanoma cell lines with low inducible levels of PD-L1 expression (PD-L1 $\left.1_{\mathrm{IND}}\right)$. We found that PD-L1 $1_{\mathrm{CON}}$ expression was associated with a reprogrammed transcriptomic state, inclusive of dedifferentiation, and active innate inflammatory pathways associated with IFN and TNF signalling and reduced oxidative phosphorylation. Overall, changes in the expression of key transcription factors were observed that drive dedifferentiation (such as the loss of MITF and SOX10), as well as key transcription factors that enhance IFN and TNF signalling and PD-L1 expression (such as IRF1, JUN, and FOSL2, in which the latter two encode an AP-1 protein complex). We additionally found that the altered expression of transcription factors and transcriptional reprogramming correlated with the PD-L1 ${ }_{\mathrm{CON}}$ mRNA expression, and with factors associated with resistance to MAPK pathway inhibitors.

\section{Materials and Methods}

\subsection{Selection and Culture of Melanoma Cell Lines}

We analysed transcriptome feature of seven PD-L1 $1_{C O N}$ lines, which includes four (CM143.pre, CM143.post, NZM9, NZM40) lines from our previous study [6] and three additional new PD-L1 $1_{\text {CON }}$ cell lines (MM127, MM595, and COLO239F) that were included in this study. These three cell lines were kindly provided by professor Glen Boyle from 
the QIMR Berghofer Medical Research Institute. For PD-L1 ${ }_{\text {IND }}$ group, we have analysed 10 cell lines, which includes six (CM138, CM150.post, CM145.pre, CM145.post, NZM22, NZM42) lines from our previous study [6] and four new cell lines (NZM12, NZM15, WM115, and WM2664). The CM138, CM145.pre, CM145.post, CM150.post, CM143.pre, and CM143.post lines were cultured in DMEM medium (Invitrogen) supplemented with 10\% foetal bovine serum (FBS) and 1\% penicillin-streptomycin. WM115 and WM2664 were cultured in Minimum Essential Medium (MEM- $\alpha$ ) (Invitrogen) supplemented with 1\% penicillin-streptomycin (Gibco, NY, USA) and 10\% FBS. NZM9, NZM40, NZM12, NZM15, and NZM42 were cultured in MEM- $\alpha$ media supplemented with $1 \%$ penicillinstreptomycin, 5\% FBS, and 0.1\% Insulin-transferrin-selenium (Roche) [13]. MM127, MM595, and COLO239F were grown in RPMI 1640 (Thermo Fisher Scientific, Waltham, MA, USA) Medium supplemented with $10 \%$ FBS and 1\% penicillin-streptomycin. All cells were grown under standard cell culture conditions $\left(5 \% \mathrm{CO}_{2}, 21 \% \mathrm{O}_{2}, 37^{\circ} \mathrm{C}\right.$, humidified atmosphere), except WM115, which were cultured at $35^{\circ} \mathrm{C}$.

\subsection{Flow Cytometry Analysis}

Fixable viability stain 450 (FVS450, BD horizon, catalog\#: 562247, clone: 29E.2A3) was used to stain dead cells in order to selectively analyse live cells. The FVS450 stain has a fluorescence emission maximum at $450 \mathrm{~nm}$. The PE anti-human CD274/PD-L1 (Biolegend, catalog\# 329706) antibody and the isotype control antibody (PE Mouse IgG2b, Biolegend, catalog\#:400314) have a maximum excitation at $575 \mathrm{~nm}$. No overlap in fluorescence emission was detected between the FVS450 and the anti-PDL1 fluorophore or isotype control antibodies. The PD-L1 expression levels of melanoma cell lines were determined using BD FACS CantoII. All analyses were performed using the Kaluza (Beckman Coulter, version 2.0, Carlsbad, CA, USA) software. Approximately 10,000 events/cells were measured for each sample. Gating strategy was used to exclude dead cells and doublet cells. The median fluorescence intensity (MFI) for the isotype control and anti-PDL1 was obtained. The MFI for PD-L1 staining was normalised for background absorbance by subtracting out the isotype fluorescence value. We used an arbitrary MFI cut-off value of $<500$ for the PD-L1 IND group and $>10,000$ for PD-L1 $1_{\text {CON }}$ group. Gene expression values confirmed that there were significantly higher levels of CD274 expression in the PD-L1 ${ }_{C O N}$ group, with an increased $\log _{2}$ fold change of 7.2. For IFN- $\gamma$ induction of PD-L1, between 50,000 to 100,000 cells were seeded in a single well of a 24-well plate overnight with $1 \mathrm{~mL}$ of media. For each sample, around 10 wells were seeded in order to obtain a total amount of between 500,000 to 1 million cells. The following day, the media was removed and fresh media with IFN- $\gamma$ (final concentration of $100 \mathrm{ng} / \mathrm{mL}$, prospec, catalog\#: CYT-206) was added to the cells. After 1 day of IFN- $\gamma$ induction, flow cytometry was used to assess PD-L1 expression.

\subsection{RNA Extraction and Reverse Transcription}

Total RNA was isolated from melanoma cell lines using the RNeasy Mini Kit (Qiagen, catalog\#:74106) following the protocol manual. This involved cell lysis, homogenisation of the lysate using the QIAshredder (Qiagen, catalog\#:79656), and using a spin column to selectively purify RNA. DNase (RNase-Free DNase Set, Qiagen, catalog\#:79254) was used to degrade DNA during the extraction as outlined in the RNeasy Mini Handbook. Quality control was first performed on the Nanodrop 2000 Spectrophotometer (Thermo Fisher Scientific) to assess the RNA purity using the ratio of absorbance at 260 to $280 \mathrm{~nm}$ higher than 1.8. The RNA integrity was assessed using the Agilent Bioanalyzer 2100 with the RNA integrity number (RIN) higher than nine. Reverse transcription from RNA to complementary DNA (cDNA) was performed using the High-Capacity cDNA Reverse Transcription Kit (Applied Biosystems, catalog\#:4368814).

\subsection{Additional Cell Line Cohorts and TCGA Data for Melanoma}

Melanoma cell line gene expression data was obtained from external sources to validate our results. The getGEO function from the GEOquery package was used to download 
four gene expression datasets with a total of 175 melanoma cell lines. The datasets included: GSE7127 $(n=63)$ [14], GSE4843 $(n=45)$ [15], GSE61544 $(n=13)$ [16], GSE80829 $(n=54)$ [17]. Normalisation was performed where the unprocessed microarray data or raw count RNA-seq matrix was available. For GSE7127 (Affymetrix U133 Plus 2 microarray platform), the data was normalised using Robust Multichip Average (RMA, from the affy package). For GSE61544, the raw count data was downloaded and normalised using TMM (edgeR package) [18]. For GSE4843 the normalized data (MAS5.0 normalization) and GSE80829 (FPKM using conditional quantile normalization) data was downloaded used for analysis. RNA-seq data (FPKM) of melanoma cell lines prior to and following acquired resistance upon treatment with MAPK inhibitors as well as patient tumours that were on MAPKi treatment (4 weeks) was obtained from GSE75313 [5]. Patient tumour RNA-seq data containing baseline, and after tumour progression, was obtained from GSE65186 (FPKM normalised gene expression matrix) $(n=70)$ [19]. RNA-seq data (FPKM normalised gene expression matrix) of melanoma cell lines $(n=8)$, treated with IFN- $\gamma$ for 2 to 5 weeks or TNF for 3 days, was acquired from GSE152755 [20].

\subsection{TCGA SKCM Data Download}

The RNA-seq count matrix was downloaded from the harmonized TCGA SKCM dataset (GRCh38). This was done using the RTCGAbiolinks package in $\mathrm{R}$ which downloads the data from the Genomic Data Commons (GDC) database [21]. The GDCquery function was used with the parameters: project = "TCGA-SKCM", data.category = "Transcriptome Profiling", data.type = "Gene Expression Quantification", workflow.type = "HTSeqCounts"). The TMM method was used to normalise the count matrix.

\subsection{Generation and Processing of Transcriptome Data}

RNA-seq processing for PD-L1 $1_{\text {IND }}$ and PD-L1 ${ }_{\text {CON }}$ melanoma cell lines was performed with poly-A-tail selection, paired-end reads, read length of $2 \times 100 \mathrm{bps}$, and a total of 40 million reads. Adaptor trimming was done using cleanadaptors from the DMAP package $[22,23]$. The RNA-Seq design and analysis that was used in this work has been described in detail previously [24]. Pseudoalignment methods such as Kallisto and Salmon were found to outperform other alignment methods when measuring lncRNA expression abundance [25]. Therefore, we mapped our reads to the hg38 reference genome using Kallisto (version 0.44.0) [26]. Each sample was run with 100 bootstraps and with the bias argument to correct for potential sequence-based bias. Annotations were acquired from GENECODE (Release 28 GRCh38.p12), which entailed nucleotide sequences of all transcripts (protein-coding and lncRNA transcripts) on the reference chromosomes. Tximport was used to import the kallisto gene-level counts data into R [27].

\subsection{Statistical Analysis}

Following filtering our genes with low counts (lower than 1 in at least seven samples), the TMM method was used to normalise for sequencing depth and RNA composition bias using the edgeR package [18]. Differential expression analysis was performed using edgeR quasi-likelihood method [28]. A False Discovery Rate (FDR) adjusted $p$ value threshold of 0.05 was used to call significant genes. Gene Set Enrichment Analysis (GSEA) was performed using gene sets available in the Broad Institute Molecular Signatures Database (MsigDB) which included H1 (hallmarK), C2 (curated), and C5 (gene ontology) [29]. CAMERA test was performed as available from the edgeR package and a FDR adjusted $5 \times 10^{-5} p$ value was used as the statistically significant threshold [30]. For generating a gene-set score for each sample, single sample GSEA (ssGSEA) [31] was used from the GSVA Bioconductor R package [32]. The IFN score, TNF score, differentiation score, and oxidative phosphorylation score were obtained from MSigDB from the "MOSERLE_IFNA_RESPONSE", "PHONG_TNF_TARGETS_UP", “GO_MELANOCYTE_DIFFERENTIATION", and “KEGG_OXIDATIVE_PHOSPHORYLATION" gene sets, respectively. The viral mimicry score was self-curated from published arti- 
cles $[33,34]$ and included DDX58, DDX41, IFIH1, OASL, IRF7, IRF1, ISG15, MAVS, IFI27, IFI44, IFI44L, and IFI16. Differentiation signature genes from Tsoi and colleagues [17] (from Table S3) were acquired to further evaluate the dedifferentiation signature in our melanoma cell lines. These genes included 6 groups, in order, from least differentiated to the most differentiated: (1) Undifferentiated, (2) Undifferentiated-Neural crest-like, (3) Neural crestlike, (4) Transitory, (5) Transitory-Melanocytic, (6) Melanocytic. Z-scores were generated for each gene and unsupervised hierarchical clustering was performed. To infer cytotoxic immune activity from gene expression data, the CYT-score was calculated by finding the geometric mean from GZMA (granzyme A) and PRF1 (perforin) expression values [35]. The absolute abundance of eight immune and two stromal cell populations were estimated from normalised RNA-seq data using MCPcounter [36]. R codes to calculate the moving average and to generate figures were acquired from Riesenberg and colleagues [37].

\section{Results}

3.1. PD-L1 $\mathrm{CON}$ Melanoma Cell Lines (High PD-L1 Group) Have a Distinct Gene Expression Profile Compared to PD-L1 IND Melanoma Cell Lines (Low PD-L1 Group)

As we have previously suggested [6], melanomas can be categorized into four subgroups based on high or low levels of tumour-associated PD-L1 protein expression, and the presence or absence of TILs in melanoma tissue. In this study, we defined these subgroups as TIL+/PDL1+ (group 1), TIL-/PDL1- (group 2), TIL+/PDL1- (group 3), and TIL-/PDL1+ (group 4) (Figure 1A). Factors leading to PD-L1 expression in melanoma tumour tissues are complicated by the fact that cytokines secreted from TILs frequently induce PD-L1 expression in tumour cells, which masks whether cytokine-independent, tumour cellintrinsic PD-L1 expression (i.e., PD-L1 constitutive expression, or PD-L1 $\mathrm{CON}_{\mathrm{N}}$ ) has occurred. Melanoma cell lines grown in vitro do not have TILs present, and yet some melanoma cell lines constitutively express high PD-L1 levels, while other melanoma cell lines express low baseline levels of PD-L1, but the latter subgroup can be induced to express higher PD-L1 levels with IFN $\gamma$ stimulation. Therefore, we used melanoma cell lines to begin our investigation, as they lack TILs, and high levels of constitutive PD-L1 expression in these cell lines consequently represent tumour cell-intrinsic mechanisms. Assignment of a panel of melanoma cell lines to the high and low PD-L1 expression groups was accomplished by characterizing cell surface PD-L1 protein levels and mRNA expression levels, which were assessed using flow cytometry and RNA-Seq [38] (Figure S1A,B, Table S1). We found that, as opposed to a continuum from low to high of CD274 (PD-L1 mRNA) expression, the $C D 274$ expression in the melanoma cell lines was either at a low level (yet inducible, hence PDL1 $\left.1_{\text {IND }}\right)$, or at a constitutively high level (PDL1 $\left.1_{\mathrm{CON}}\right)$. The CD274 mRNA levels (as quantified by RNA-Seq) strongly correlated with the PD-L1 surface protein expression levels (Pearson $R=0.92, p$ value $=1.4 \times 10^{-7}$ Figure S1C) in the analysed 17 cell lines (seven PD-L1 $1_{\mathrm{CON}}$ cell lines and ten PD-L1 $1_{\mathrm{IND}}$ cell lines). Given that melanoma cell lines and patient tumours occasionally exhibit dysfunctional IFN signalling due to genetic defects [39-43], we additionally verified, by PD-L1 upregulation upon IFN $\gamma$ treatment, that ten low PD-L1 IND cell lines in our panel were inducible, which confirmed them as being able to respond to IFN $\gamma$ induction (Figure S1D), while PD-L1 ${ }_{\mathrm{CON}}$ weren't inducible [38]. 
A

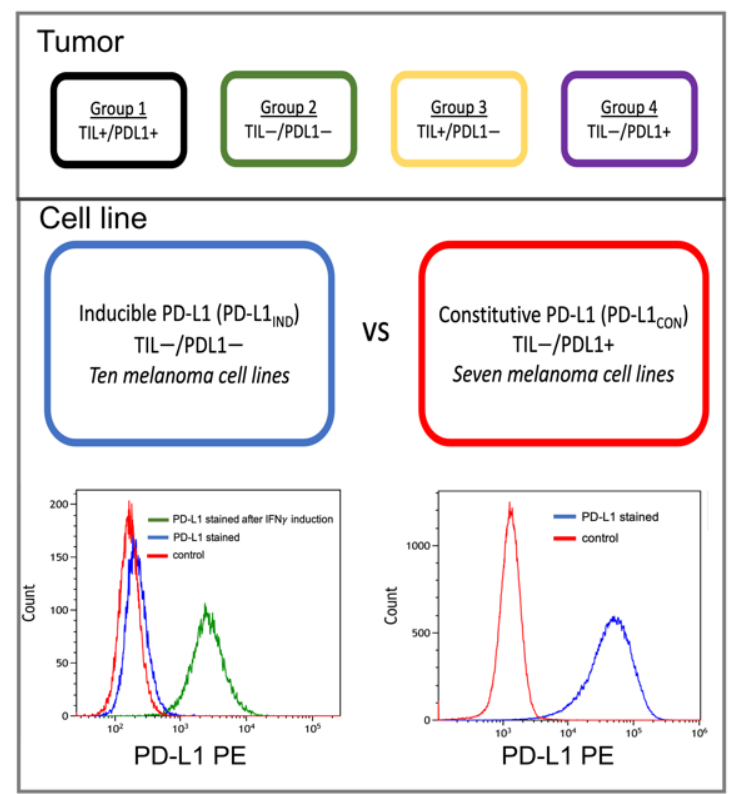

B

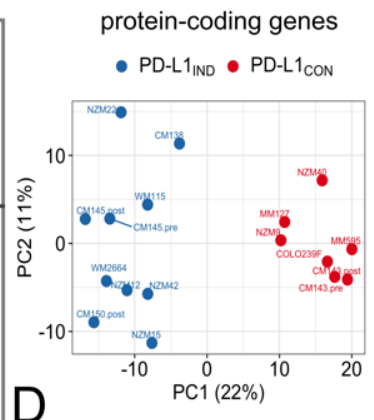

protein-coding genes

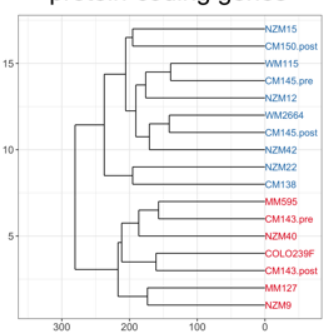

C

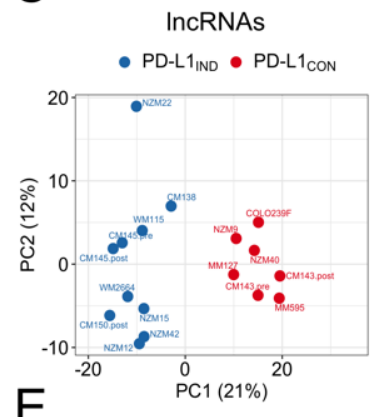

E

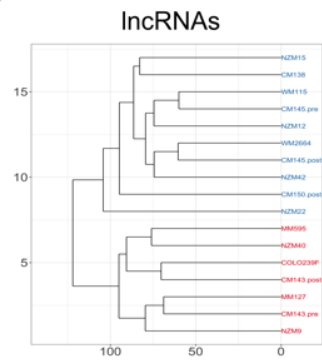

$\mathrm{F}$

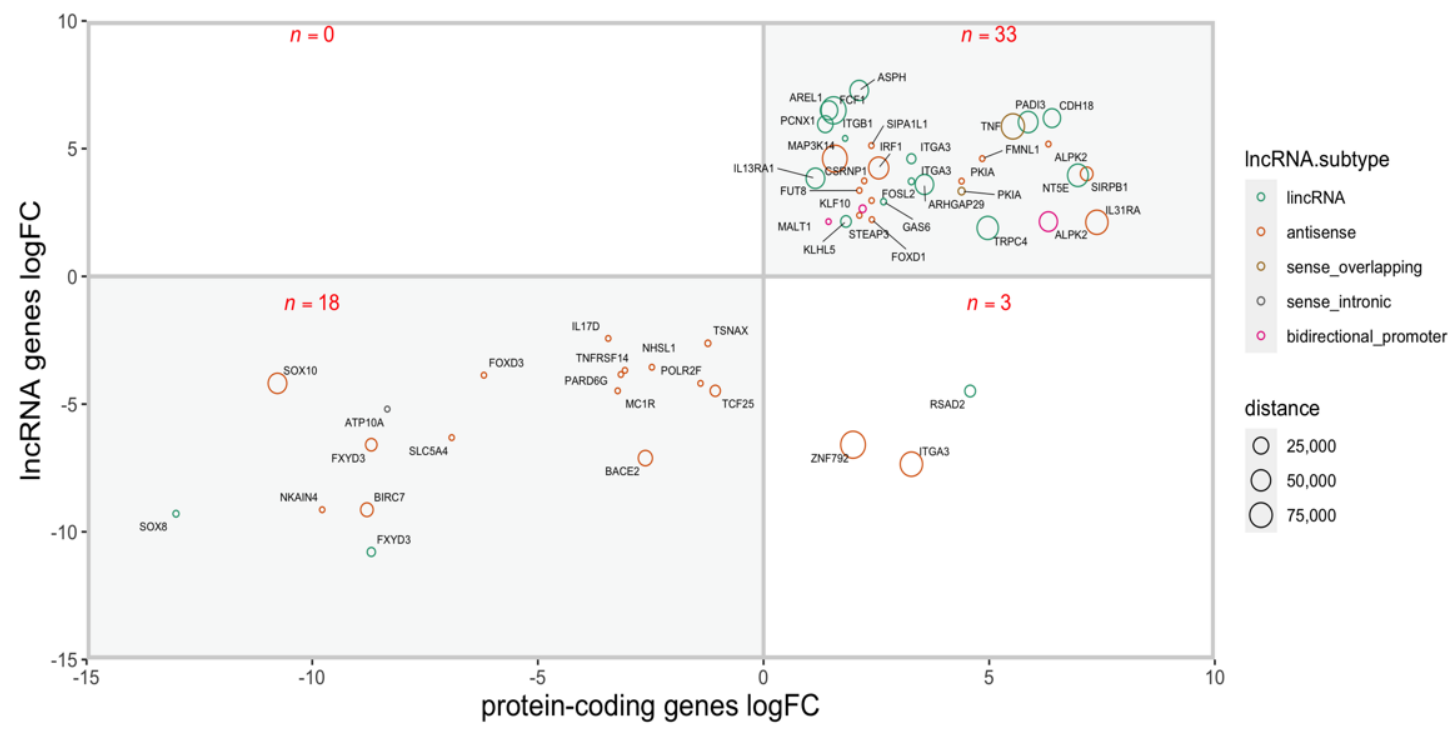

Figure 1. The PD-L1 $\mathrm{CON}$ group harbours distinct global mRNA and long non-coding RNA expression profiles compared to the PD-L1 $1_{\mathrm{IND}}$ group. (A) A schematic diagram showing the four subgroups of melanoma tumours based on the levels of tumour infiltrative lymphocytes and PD-L1 protein expression [6]. We used melanoma cell lines and compared transcriptomes between cell lines corresponding to the low PD-L1 (group 2: TIL-/PDL1- or PD-L1 $1_{\mathrm{IND}}$ ) and high PD-L1

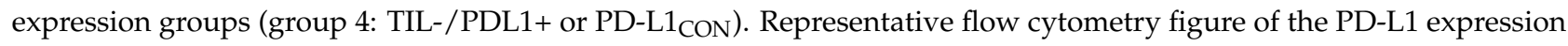
levels in each of the cell lines, representing the two groups are shown below. The flow cytometry figure shows isotype control (red), PD-L1 stained (blue), and PD-L1 stained following 24-hour IFN $\gamma$ induction (green). The x-axis represents PD-L1 expression levels $\left(\log _{2}\right)$ and y-axis represents cell counts. (B,C) Principal component analysis (PCA). Shows PC1 (x-axis) and PC2 (y-axis) for the PD-L1 ${ }_{\mathrm{IND}}$ samples and the PD-L1 $1_{\mathrm{CON}}$ samples using the top 500 protein-coding genes (B) or lncRNAs (C) with the highest variance. (D,E) Unsupervised hierarchical clustering of PD-L1 $1_{C O N}$ and PD-L1 $1_{\text {IND }}$ melanoma cell lines using either expression of all protein-coding genes, or of lncRNAs. (F) The significantly differentially expressed mRNA-IncRNA gene pairs that are located within 100 kilobases to one another are shown. The $x$-axis and the $y$-axis show the protein-coding gene and lncRNA expression fold change $\left(\log _{2}\right)$, respectively. Only the protein-coding gene names are shown. The distance between the mRNA-lncRNA gene pair is represented by the size of the circle, and the lncRNA subtype is shown by the colours. 
Principal component analysis of the top 500 protein coding (Figure 1B) and long non-coding RNA (Figure 1C) genes with the highest variance segregated the cell lines into two distinct clusters. Unsupervised hierarchical clustering showed the same result with a clear segregation of the two groups (Figure 1D,E), which suggests there is a distinct transcriptional state between PD-L1 $1_{\mathrm{CON}}$ and PD-L1 IND melanoma subgroups. Differential expression analysis identified 462 upregulated and 298 downregulated protein coding genes in the PD-L1 $1_{\text {CON }}$ group, compared to the PD-L1 IND group (FDR adjusted $p$ value $<0.05$ ) (Table S2). As expected, CD274 expression was significantly increased in the PD-L1 $\mathrm{CON}$ group (FDR adjusted $p$ value $=0.001, \log _{2}$ fold-change $=7.1$, Figure S2A,C). PDCD1LG2 (which encodes the PD-L2 protein, and is also located in the same chromosome location as CD274, i.e., 9p24.1) was also significantly upregulated in the PD-L1 $1_{C O N}$ group (FDR adjusted $p$ value $=0.03, \log _{2} \mathrm{FC}=5.0$, Figure S2A,D). For the long non-coding RNA (lncRNA) analysis, we identified 106 upregulated and 71 downregulated lncRNA in PD-L1 $1_{\mathrm{CON}}$ lines compared to the PD-L1 $1_{\mathrm{IND}}$ lines (Figure S2B, Table S2). The upregulated lncRNAs included 75 lincRNAs, 26 antisense, 3 bidirectional promoter and 2 sense overlapping lncRNAs, while the downregulated lncRNAs included 37 lincRNAs, 31 antisense, and 3 sense intronic lncRNAs. Next, given that lncRNAs can regulate gene expression via cis mechanisms, we assessed all differentially expressed lncRNAs with loci mapped adjacent to (within $100 \mathrm{~kb}$ ) a differentially expressed protein-coding gene, which identified 54 mRNA-lncRNA pairs. 51 out of the $54(94.4 \%)$ mRNA-lncRNA pairs showed expression changes in the same direction (either up or down), with 33 pairs being upregulated (top-right quadrant, Figure 1F), while 18 pairs showing downregulation (bottom-left quadrant, Figure $1 \mathrm{~F}$ in the PD-L1 $1_{\mathrm{CON}}$ lines compared to the PD-L1 IND group. These results suggest coordinated expression changes for the mRNA and lncRNA expression. Overall, PD-L1 $1_{\mathrm{CON}}$ cell lines had a distinct expression profile for protein-coding genes and lncRNAs, and moreover the vast majority of the differentially expressed mRNAs and lncRNAs located in genomic proximity to each other were altered in the same direction.

\subsection{PD-L1 $\mathrm{CON}$ Cell Lines Exhibit a Distinct Transcriptome That Represents a State of Dedifferentiation, Enhanced IFN, and TNF Signalling Pathways and Reduced Oxidative Phosphorylation}

To determine which biological processes are altered in the PD-L1 CON $_{\text {coll lines com- }}$ pared to PD-L1 IND cell lines, we performed gene-set enrichment analysis using CAMERA [30] and C2, C5, and $\mathrm{H}$ gene set collections from the Molecular Signature Database (MSigDB) [29]. Thirty-seven gene sets were found to be significantly altered (FDR adjusted $p$ value threshold of $5 \times 10^{-5}$ ). Genes involved in interferon-alpha (IFN- $\alpha$ ) and tumour necrosis factor (TNF) signalling were among the most significantly upregulated processes in the PD-L1 1 CON group ((FDR adjusted $p$ value $=3.0 \times 10^{-10}$ (MOSERLE_IFNA_RESPONSE) and $1.1 \times 10^{-9}($ PHONG_TNF_TARGETS_UP), Figure 2A and Figure S3A). Given that the PD-L1 $1_{\mathrm{CON}}$ cell lines do not harbour immune cells, this suggests that the IFN and TNF signalling could be activated via the expression of dsRNA derived from endogenous retroviral elements (ERVs). Expression of ERVs can trigger dsRNA sensors and a downstream signalling cascade of interferon response genes (also referred to as a viral mimicry response) $[33,34]$. Genes involved as dsRNA sensors, as well as the viral mimicry response genes, were also highly upregulated in PD-L1 $1_{C O N}$ group (FDR adjusted $p$ value $=7.5 \times 10^{-4}$ and $2.1 \times 10^{-4}$, respectively) (Figure S3A). These findings suggest that PD-L1 $1_{\mathrm{CON}}$ samples may have enhanced activation of viral mimicry pathways. This is also supported in our previous findings, in that ERV genomic regions were hypomethylated in PD-L1 $1_{\mathrm{CON}}$ melanoma cells [6], and were a characteristic feature associated with PD-L1 expression. The downregulated genes in the PD-L1 $1_{\mathrm{CON}}$ samples were significantly enriched for genes involved in melanocyte differentiation (FDR adjusted $p$ value $=2.7 \times 10^{-6}$, Figure 2A and Figure S3A), suggestive of a dedifferentiated state. Recently, Tsoi and colleagues found that melanomas can exhibit transcriptomic states that are coupled to a differentiation trajectory, which consists of four progressive steps [17], corresponding to (1) undifferentiated, (2) neural crest-like, (3) transitory, and (4) melanocytic. Unsupervised 
hierarchical clustering of the cell lines based on these melanoma differentiation genes confirmed that the PD-L1 $1_{\mathrm{CON}}$ cell lines were enriched for genes characteristic of the undifferentiated state (Figure $2 \mathrm{~B}$ ). The PD-L1 IND cell lines were further clustered into two subgroups from their transcriptomic profiles, with one group corresponding to a neural crest-like gene signature, and the other group to a melanocytic state. Moreover, there was a downregulation of genes involved in oxidative phosphorylation in the PD-L1 ${ }_{\mathrm{CON}}$ group, suggesting metabolic reprogramming had occurred (FDR adjusted $p$ value $=7.2 \times 10^{-8}$ (KEGG_OXIDATIVE_PHOSPHORYLATION)) (Figure 2B and Figure S3A). Given that dedifferentiation is closely associated with enhanced invasiveness we investigated whether the PD-L1 $1_{\mathrm{CON}}$ samples were enriched for an invasive gene expression profile. Using two independent invasive gene expression gene sets $[44,45]$, we found PD-L1CON cell lines had higher levels of expression of genes involved in melanoma invasion (Figure S4A,B) although three PD-L1 IND cell lines (NZM22, WM115, CM145.pre) had weak expression levels of invasive genes, whereas CM138 clustered more closely to the PD-L1 $1_{\text {CON }}$ cell lines.

3.3. Validation of Upregulated IFN and TNF Pathways, and Downregulated Differentiation and Oxidative Phosphorylation Pathways in Association with Constitutive CD274 Expression in Melanoma Cell Lines

To validate the gene expression signature of an upregulated IFN and TNF pathway, and downregulation of differentiation and oxidative phosphorylation genes in PD- L1 1 CON melanoma cells, we utilised four external gene expression datasets containing a total of 175 melanoma cell lines. A score was generated for the 462 upregulated and 298 downregulated genes in the PD-L1 $1_{\mathrm{CON}}$ samples (referred to as the up and down PD-L1 $1_{\mathrm{CON}}$ score respectively) as well as the genes involved in IFN signalling, TNF signalling, differentiation, and oxidative phosphorylation using single sample gene set enrichment analysis (ssGSEA) [31,32]. In all four external gene expression datasets, CD274 expression was significantly positively correlated with IFN and TNF scores and negatively correlated with the differentiation score (Figure 2C). However, the oxidative phosphorylation score did not show consistent correlation with $C D 274$ expression in the external gene expression datasets (Figure S3B).

We have previously found that blocking IFN signalling in the PD-L1 $1_{\mathrm{CON}}$ samples did not alter the PD-L1 $1_{\mathrm{CON}}$ expression in two of the PD-L1 $1_{\mathrm{CON}}$ samples that were also used in this study [38], which demonstrates that PD-L1 $1_{\mathrm{CON}}$ expression is associated with a cell intrinsic regulated IFN response [6]. However, in tumours, immune cell infiltrates and their secreted cytokines are thought to be mainly responsible for extrinsically inducing IFN signalling, which in turn upregulates PD-L1 expression [9]. To understand whether immune cell independent PD-L1 $1_{\mathrm{CON}}$ expression is similar to that in tumours with an immune cell dependent PD-L1 expression, we asked whether the PD-L1 $1_{\mathrm{CON}}$ samples share an expression signature with those tumours with an elevated immune infiltration. To this end, the TCGA SKCM dataset which contains RNA-seq data for 472 tumours was analysed. A large proportion of significantly upregulated transcripts (135 out of 462 or $29 \%$ ) in the PD-L1 $1_{\text {CON }}$ samples were positively correlated (Pearson correlation value of higher than 0.25 ) with the CYTscore (Figure 2D), which is a well-established index that reflects cytolytic immune activity [35]. Moreover, the upregulated and downregulated PD-L1 $1_{\mathrm{CON}}$ genes were positively and negatively correlated, respectively, with the absolute abundance of a large range of immune subtypes as estimated by MCPcounter [36] (Figure 2D), demonstrating that PD-L1 $1_{\mathrm{CON}}$ melanoma cell lines have an immune cell independent active cytokine signalling pathway similar to melanoma tumours where immune infiltrates stimulate this pathway. 

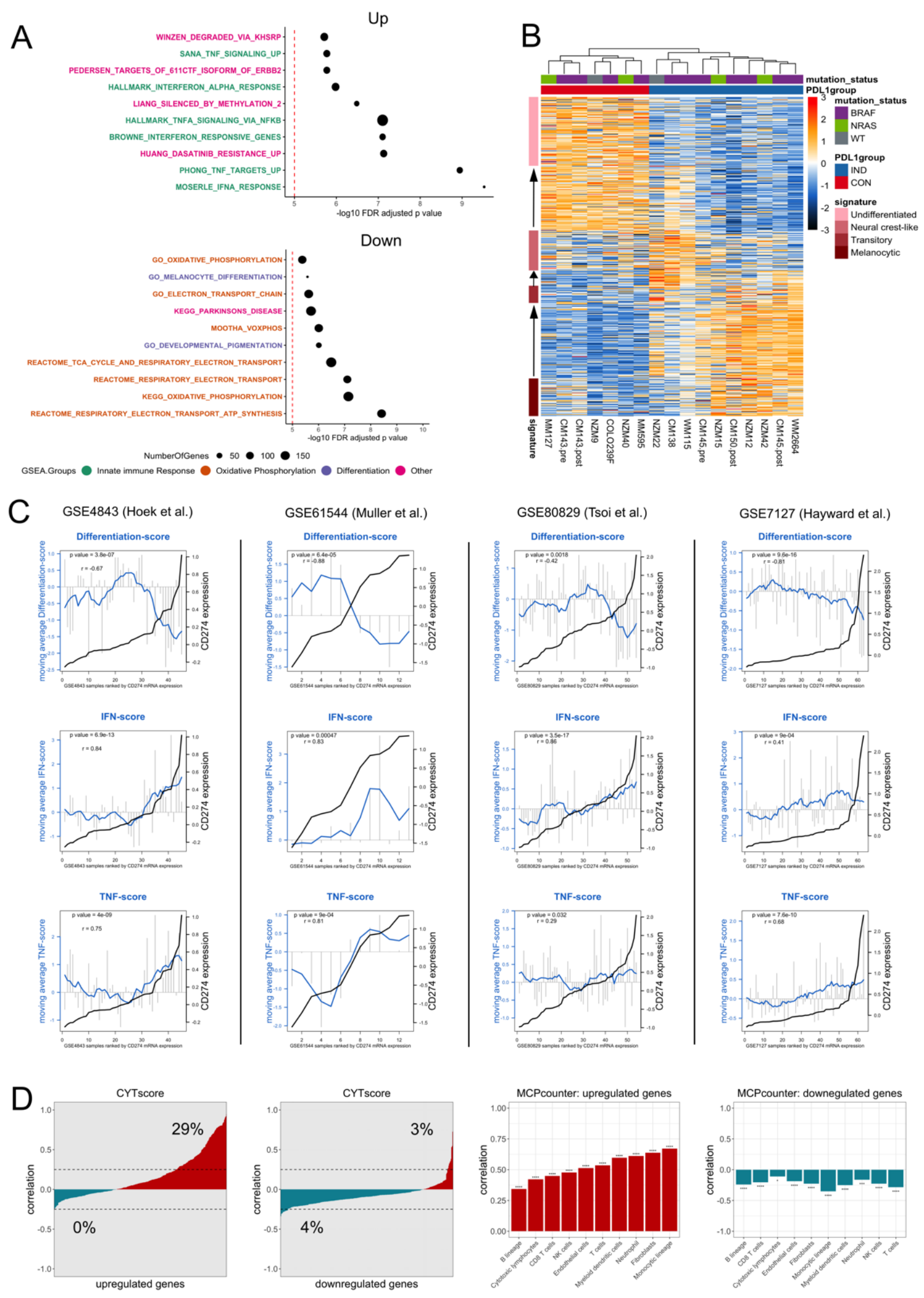

Figure 2. PD-L1 $\mathrm{CON}$ samples have a reprogrammed transcriptome inclusive of dedifferentiation, reduced oxidative phosphorylation, and an enhanced IFN signalling pathway. (A) The top 20 gene sets that were found to be differentially 
enriched using the CAMERA test are shown [30]. The $5 \times 10^{-5}$ FDR adjusted $p$ value significance threshold is shown by the dotted red line. Gene sets are categorised into those involved in the innate immune response (or cytokine signalling), melanocyte differentiation, oxidative phosphorylation, and others. (B) Heatmap showing the unsupervised clustering of the PD-L1 $1_{\mathrm{CON}}$ and PD-L1 IND melanoma cell lines according to the differentiation signature genes from Tsoi and colleagues [17]. Mutation status according to BRAF (V600), NRAS (Q61 and G13), and double wild-type is shown. (C) Correlation of CD274 expression with the IFN score, TNF score and differentiation score in the four external melanoma cell line datasets. The samples (x-axis) are ranked according to CD274 expression ( $\log _{2}$ transformed and corresponds to the right-side y-axis) and the CD274 expression level is shown by the black line. Grey vertical bars indicate the respective scores and corresponds to the left side y-axis. The moving average is represented by the blue line. The Pearson correlation between the moving average and CD274 expression is shown. (D, first and second figure) The y-axis represents the correlation value (Pearson) of the CYT score with either the upregulated ( $\mathbf{D}$, first figure) or downregulated protein-coding genes (D, second figure) in the PD-L1 CON samples. The x-axis represents all the up (462 genes) and downregulated (298 genes) protein-coding genes. The $\mathrm{x}$-axis is ranked according to lowest to highest correlation value (Pearson). (D, third and fourth figure) The y-axis represents the correlation value (Pearson) of the up $(\mathbf{D}$, third figure) and down PD-L1 CON scores (D, fourth figure) (calculated with ssGSEA using the differentially expressed protein-coding genes) with the estimated absolute abundance of various immune subtypes (as measured using the MCPcounter bioinformatic tool).

IFN gamma (IFN- $\gamma$ ) and TNF stimulation have been shown to induce both PD-L1 expression and a dedifferentiation phenotype $[20,46,47]$. To investigate whether IFN- $\gamma$ and TNF treatment can also activate the PD-L1 $\mathrm{CON}$ gene expression signature in cell lines, we analysed an independent RNA-seq dataset (GSE152755) [20] where eight melanoma cell lines were treated with either IFN- $\gamma$ for 2 to 5 weeks, or TNF for 3 days. Both IFN- $\gamma$ and TNF treatment demonstrated gene expression changes towards the PD-L1 $1_{C O N}$ signature (Figure S5A,B). It is important to note that the cytokine induced signatures in these cells are reversible upon removal of stimulation $[46,47]$, whereas in PD-L1CON samples, these signatures are not dependent on any external stimulation.

3.4. Validation of Upregulated IFN and TNF Pathways, and Downregulated Differentiation and Oxidative Phosphorylation Pathways in Association with Constitutive CD274 Expression in Melanoma Tumour Tissues

To validate the association of PD-L1 $1_{\mathrm{CON}}$ expression with active IFN and TNF signalling, and reduced differentiation and oxidative phosphorylation expression signatures in melanoma tumours, we used the SKCM TCGA dataset consisting of 458 melanoma tumour samples. As mentioned above, CD274 expression in melanoma tissues is typically stimulated by immune infiltrates rather than an intrinsic mechanism, therefore we used the significantly differentially expressed genes that were found in the PD-L1CON cell lines as a surrogate for PD-L1 $1_{\mathrm{CON}}$ expression. A large proportion of the upregulated proteincoding genes $(n=462)$ found in the PD-L1 $1_{C O N}$ samples were positively correlated (Pearson r $>0.25$ ) with CD274 expression (136 out of 462 or $29 \%$ ), IFN score (136 out of 462 or $29 \%$ ), and TNF score (213 out of 462 or $46 \%$ ). Furthermore, the upregulated protein-coding genes were negatively correlated (lower than -0.25 Pearson correlation value) with the differentiation (137 out of 462 or 30\%) and oxidative phosphorylation scores (151 out of 462 or 33\%) (Figure 3A and Figure S3C). In contrast, a large proportion of the downregulated protein-coding genes $(n=298)$ were positively correlated (Pearson $r>-0.25)$ with the differentiation-score (127 out of 298 or $43 \%$ ) and the oxidative phosphorylation score (108 out of 298 or 37\%) (Figure 3A and Figure S3C). The same pattern was also seen for the lncRNAs (Figure 3B). Collectively, these results support the finding that PD-L1 $1_{C O N}$ expression is associated with an enhanced IFN and TNF signalling, and reduced differentiation and oxidative phosphorylation. 
A
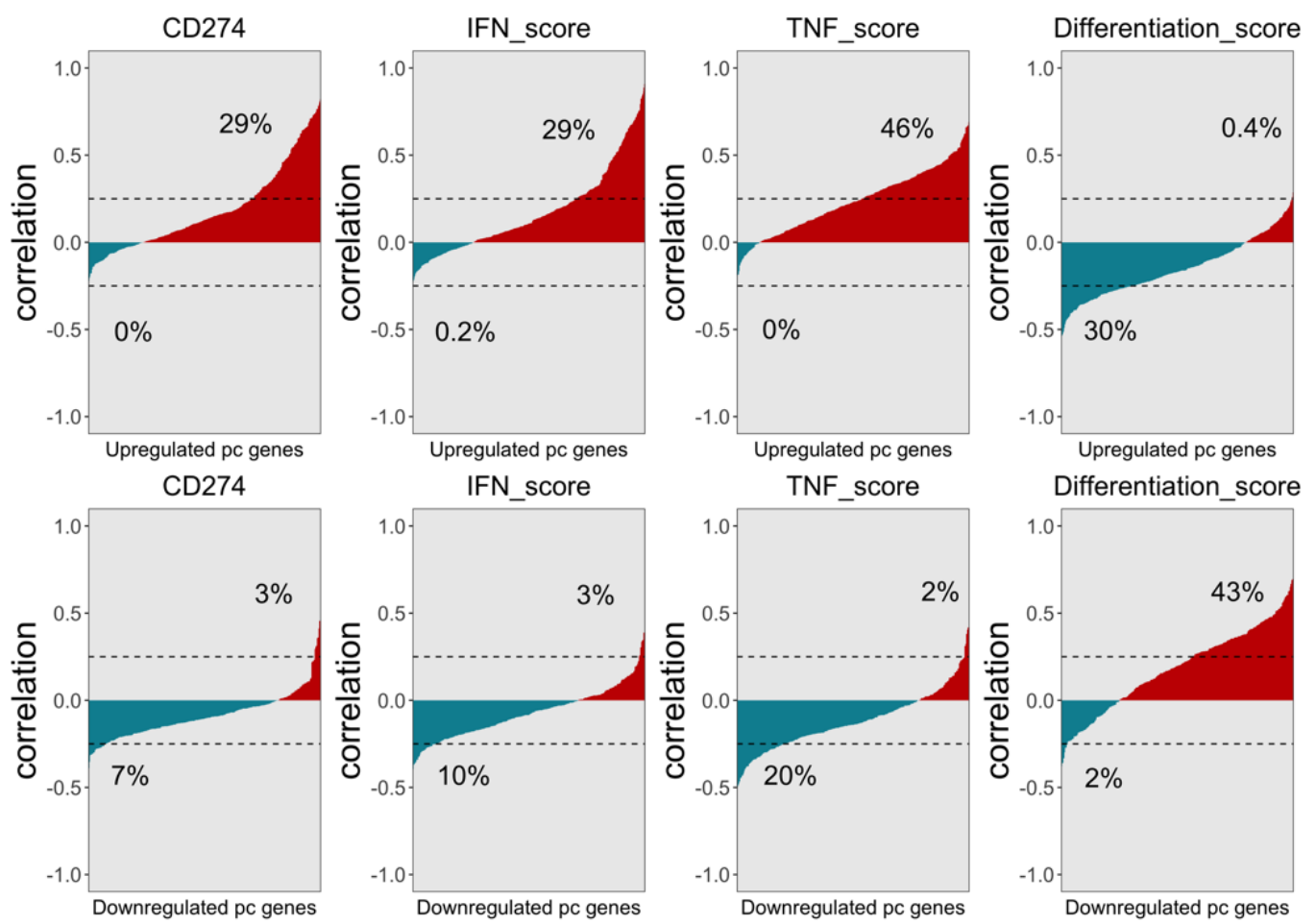

B

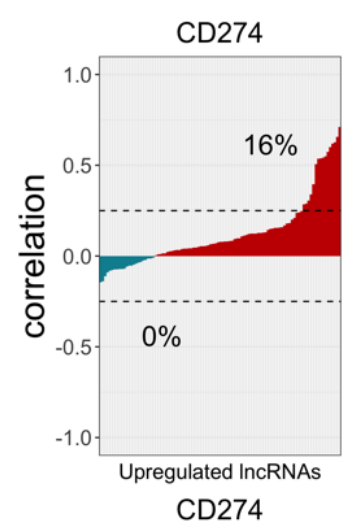

\section{long non-coding RNA}
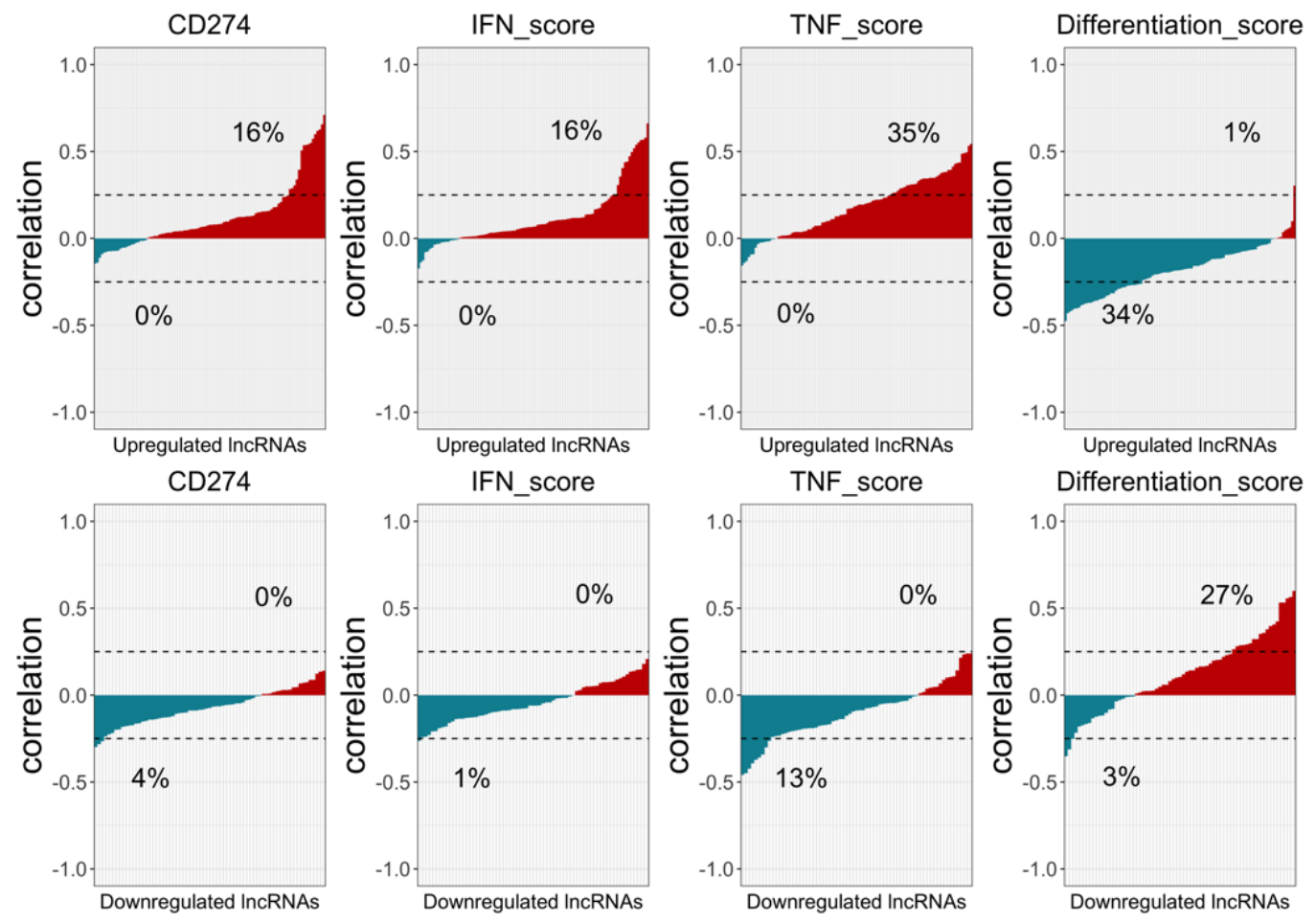

Figure 3. Protein-coding and lncRNA expression signatures of PD-L1 $1_{\mathrm{CON}}$ samples are associated with an increased IFN 
score and reduced differentiation and oxidative phosphorylation scores in the TCGA SKCM database. (A) The X-axis shows the upregulated ( $n=462)(\mathbf{A}$, top row) and downregulated $(n=298)$ (A, bottom row) protein-coding genes (A) in the PD-L1 $1_{\mathrm{CON}}$ samples. The y-axis shows the correlation (Pearson) of the protein-coding genes with CD274 expression, IFN score, differentiation score and oxidative phosphorylation score. The $x$-axis is ranked according the lowest to highest correlation. The percentage of genes higher than 0.25 or lower than -0.25 Pearson correlation value is shown. (B) The x-axis shows the upregulated $(n=106)$ and downregulated $(n=71) \operatorname{lncRNAs}$ in the PD-L1 $1_{\mathrm{CON}}$ samples. The y-axis shows the correlation (Pearson) of the lncRNAs with CD274 expression, IFN score, differentiation score and oxidative phosphorylation score. The x-axis is ranked according the lowest to highest correlation. The percentage of genes higher than 0.25 or lower than -0.25 Pearson correlation value is shown.

3.5. Lineage Specific, TNF, and IFN Associated Transcription Factors Are Differentially Expressed in the PD-L1 CON Samples

Key transcription factors (TFs) can play a role in modulating the transcriptomic program. Therefore, we investigated what type of TFs are altered in expression in the PD-L1 ${ }_{\text {CON }}$ cell lines. To this end, we analysed 1107 TF mRNAs with known DNA binding motifs [48] and identified 25 significantly upregulated TF mRNAs (out of 462 significantly upregulated genes) and 19 significantly downregulated TF mRNAs (out of 298 significantly downregulated genes) in the PD-L1 $\mathrm{CON}$ cell lines (Figure 4A). To determine whether the expression patterns of these TF mRNAs could be generalised to melanoma, we analysed the four external gene expression datasets and the TCGA SKCM RNA-seq dataset in the TF context. The 25 upregulated TF and 19 downregulated TFs were predominantly positively and negatively correlated with CD274 expression in all five datasets (Figure 4B). Furthermore, we identified POU2F2, IRF1, and FOSL2 as the most highly correlated TFs with CD274 expression in melanoma across all five datasets (Figure 4C). IRF1 and FOSL2 have known roles in upregulating PD-L1 expression [8,49,50]. In contrast, SOX10, RXRG, and SOX5 had the lowest correlation with CD274 expression amongst all TFs investigated (Figure 4C). SOX10 and MITF are key transcription factors that regulate the development of melanocytes. SOX10 expression showed a near complete loss $\left(\log _{2} \mathrm{FC}=-10.7\right.$, FDR adjusted $p$ value $=0.0003$ ) and MITF expression was significantly reduced in expression $\left(\log _{2} \mathrm{FC}=-3.8\right.$, FDR adjusted $p$ value $\left.=0.04\right)$ in the PD-L1 $1_{\mathrm{CON}}$ cell lines (Figure S6E,F). The upregulation of these TF expression further supports our observation that the PD-L1 $1_{\mathrm{CON}}$ melanoma subgroup is associated with substantial transcriptomic reprogramming.

Additionally, we identified all differentially expressed lncRNAs whose loci were located in close proximity/adjacent to differentially expressed TF mRNAs (within $100 \mathrm{~kb}$ of each other). Correspondingly, eight TF mRNA and lncRNA differentially expressed pairs were found (Figure 4D). Out of these eight TFs, four were identified to be closely associated with CD274 expression in the five external datasets. This included AL031587.1 antisense lncRNA near SOX10 (distance of 44,743 bp), the AC116366.2 intergenic lncRNA near IRF1 (distance of 54,895 bp), and FLJ31356 antisense lncRNA near FOSL2 (distance of $0 \mathrm{bp}$, as they overlap) (Figure 4D).

3.6. The PD-L1 $1_{C O N}$ Expression Signature Is Associated with Transriptomic Reprogramming, and Correlates with MAPK Inhibitor Resistance in Melanoma Cell Lines

Key transcriptional changes that were shown to drive acquired resistance include increased expression of $c M E T$, reduced expression of $L E F 1$, and an enrichment of YAP1 signature genes [19]. PD-L1 $1_{\mathrm{CON}}$ cell lines had increased expression of $c M E T\left(\log _{2} \mathrm{FC}=2.6\right.$, FDR adjusted $p$ value $=0.07)$, reduced expression of LEF1 $\left(\log _{2} \mathrm{FC}=-3.2\right.$, FDR adjusted $p$ value $=0.017$ ), and enrichment of YAP1 signature genes (t.test $p$ value $=0.012$ ) (Figure S6B-D). Other gene expression changes driving drug resistance include reduced levels of MITF and SOX10, which were both downregulated in the PD-L1 ${ }_{\mathrm{CON}}$ cells. Moreover, consistent with increased expression of Receptor Tyrosine Kinases (RTK), which are associated with drug resistance, we found a large number of RTK genes were highly expressed in the PD-L1 $\mathrm{CON}$ cell lines including EGFR $\left(\log _{2} \mathrm{FC}=4.2\right.$, FDR adjusted $p$ value $\left.=0.054\right)$, PDGFRB $\left(\log _{2} \mathrm{FC}=3.6\right.$, FDR adjusted $p$ value $\left.=0.03\right)$, and $A X L\left(\log _{2} \mathrm{FC}=2.5\right.$, FDR adjusted 
$p$ value $=0.34)\left(\right.$ Figure S6A,G-I). These data support the notion that PD-L1 $1_{\mathrm{CON}}$ cell lines have a transcriptional profile corresponding to a drug resistant phenotype.

A

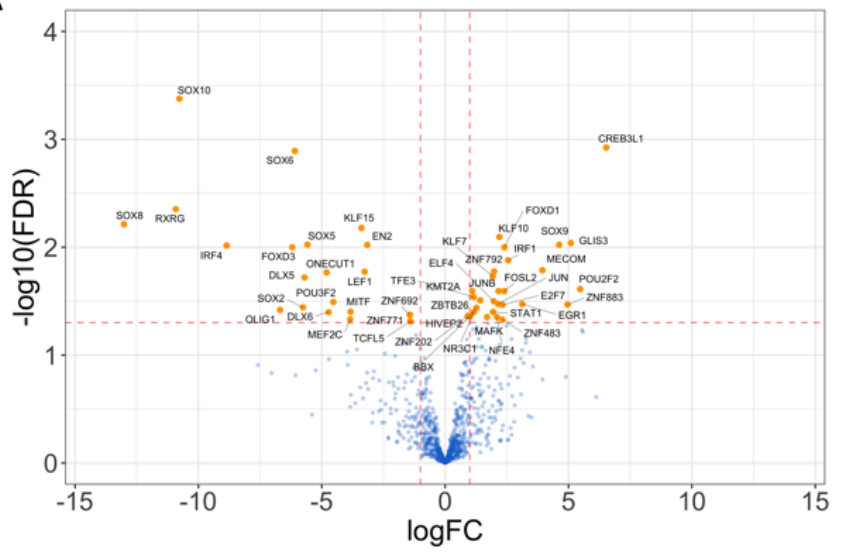

B

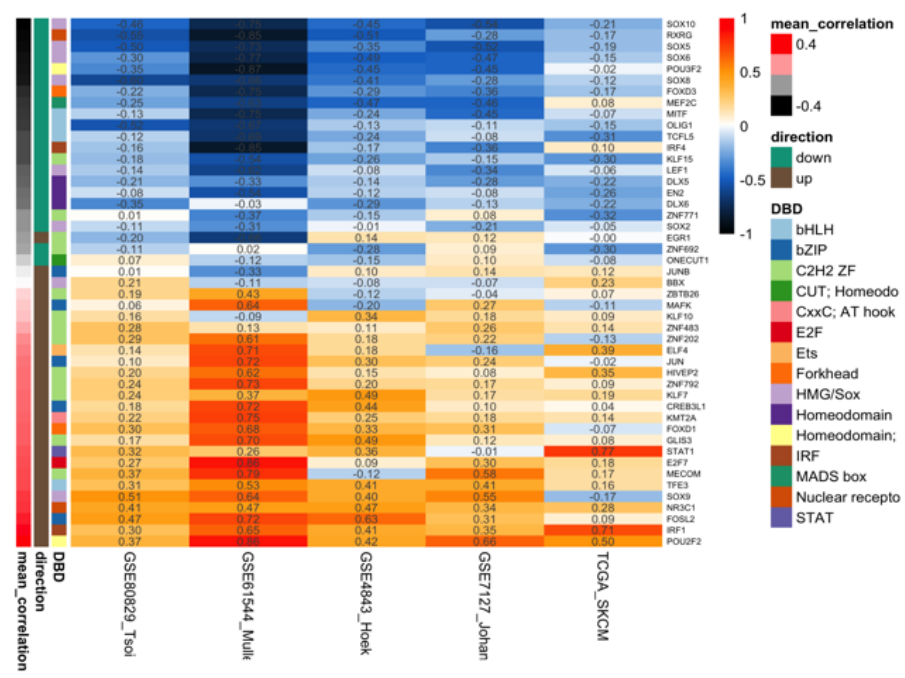

$\mathrm{D}$

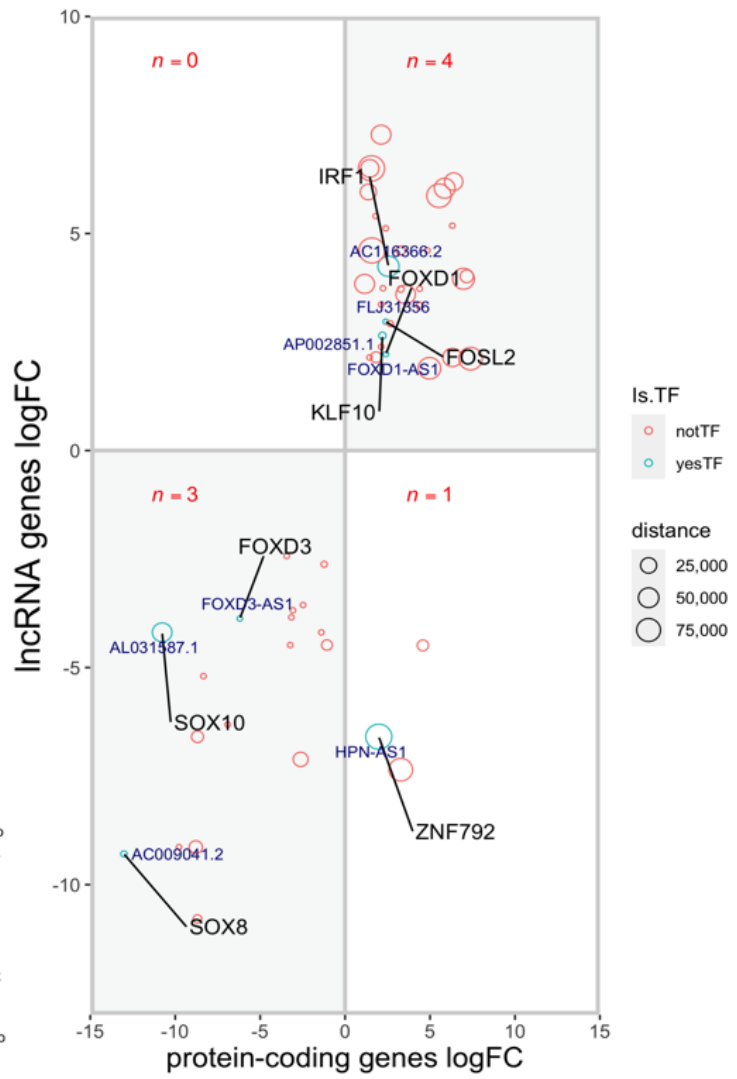

C

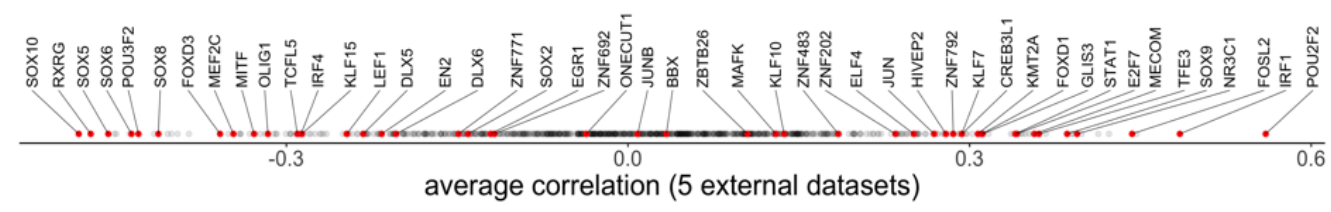

Figure 4. Correlation of lineage-specific and immunity-associated transcription factor mRNAs with PD-L1 expression in melanoma and association with long non-coding RNAs. (A) Volcano plot showing all the differentially expressed TFs in the PD-L1 $1_{\text {CON }}$ group. The 25 and 19 significantly upregulated and downregulated TF mRNAs in the PD-L1 CON samples are shown, respectively. The $x$-axis represents $\log _{2}$ fold change and the $y$-axis represents $-\log _{10}$ FDR adjusted $p$ value. The red dashed line represent the FDR adjusted $0.05 p$ value (y-axis) and the $1 \log _{2}$ fold change (x-axis). (B) Heatmap showing the correlation value (Pearsons) of the 25 up and 19 down regulated TFs with CD274 expression. Rows represent the TFs and are ordered according to the lowest to highest mean correlation with CD274 expression across the four melanoma cell line datasets and the TCGA SKCM RNA-seq dataset. (C) Correlation was calculated between CD274 expression and all TF $(n=1107)$ across the five gene expression datasets and the TF were ordered from highest to lowest mean average correlation across the five datasets. The direction of the 25 upregulated and 19 downregulated TFs are shown by the direction sidebar. (D) The significantly differentially expressed TFs to lncRNA pairs that are located within 100 kilobases to each other are shown. The $x$-axis and the $y$-axis show the TF mRNA and lncRNA expression fold-change $\left(\log _{2}\right)$, respectively. The distance between the TF mRNA and lncRNA gene are shown by the size of the circle. 
CD274 expression was found to be cell-intrinsically increased upon development of resistance to MAPK inhibitors, when accompanied by transcriptomic reprogramming, but not mediated by BRAF mutations (splicing or amplification) reactivating the MAPK pathway [5]. We utilised this dataset to assess our PD-L1 ${ }_{\mathrm{CON}}$ gene expression signature in the context of development of resistance. In cell lines where resistance co-occurred with transcriptomic reprogramming, there was an increase of $C D 274$ expression as well as IFN, TNF, and "up" PD-L1 $1_{\mathrm{CON}}$ scores (Figure 5B), and a corresponding reduction in differentiation, oxidative phosphorylation, MPAS, and "down" PD-L1 $1_{\mathrm{CON}}$ scores. Moreover, we found that TFs associated with PD-L1 $\mathrm{CON}$ expression, and involved in IFN signalling and TNF signalling (IRF1, JUN, and FOSL2), were all upregulated (Figure 5E), except for IRF4, which was downregulated in these cell lines. Consistently, IRF4 was the only TF involved in IFN and TNF signalling that was also downregulated in PD-L1 $1_{\mathrm{CON}}$ cell lines (Figure 5C). In addition, TFs involved in melanocyte differentiation (including SOX10, $M I T F$, and LEF1) were downregulated in cell lines that the authors had associated with the development of drug resistance (Figure 5E). In contrast, in cell lines where resistance was mediated by reactivation of the MAPK signalling pathway (involving BRAF splicing or amplification), only minimal changes in the expression of CD274 and the PD-L1 $1_{\mathrm{CON}}$ related biological processes (including IFN signalling, TNF signalling, differentiation, and oxidative phosphorylation) were observed (Figure 5A). Additionally, the expression of TFs involved in IFN or TNF signalling, or melanocyte differentiation were unaltered when the drug resistance was mediated by BRAF splicing or amplification mutations (Figure 5D).

\subsection{Transcriptomic Changes, including PD-L1 $1_{C O N}$ Expression, Occur at Defined Stages during the Development of Drug Resistance}

To investigate the timing of when PD-L1 expression changes and biological processes may occur during drug treatment, we investigated RNA-Seq data derived from two melanoma cell lines (M229 and M238) [5], which had been treated with an MAPK inhibitor, and for which RNA-Seq analysis was carried out at different time points between the initial resistance, generation of drug tolerance, and finally permanent acquired resistance. After two days of BRAFi treatment (BRAFi2D), the time points included; at the Drug Tolerant Persisters (DTP) stage where small subpopulation of persisting cells remained; at the Drug Tolerant Proliferative Persisters (DTPP) stage where proliferation was regained; and finally at the Single Drug Resistant (SDR) stage, which is a permanent resistant state to BRAFi (months to years of drug treatment). There was little or no PD-L1 $1_{\mathrm{CON}}$ "up" score after 2 days of BRAFi treatment (Figure $5 \mathrm{~F}$ ), suggesting that the $\mathrm{PD}-\mathrm{L} 1_{\mathrm{CON}}$ signature is not up-regulated due to immediate on-target effects of treatment with BRAFi. CD274 expression was slightly decreased at the DTP stage, however increased at the proliferative phase (DTPP) and was further increased at the SDR phase (Figure 5F). TNF, IFN, and PD-L1 $1_{C O N}$ "up" scores were largely increased at the DTPP stage, whereas dedifferentiation and PD-L1 $1_{\mathrm{CON}}$ "down" scores were largely decreased at the SDR stage. This suggested that innate IFN and TNF signalling responses precede dedifferentiation. Further analysis revealed that JUN and FOSL2, which encode proteins that are part of the AP-1 complex, and are part of the TNF signalling pathway, were increased relatively early after two days of BRAFi treatment and were maintained until acquired resistance developed, whereas IRF1, which can drive PD-L1 expression, increased at the DTPP stage when CD274 was also overexpressed (Figure 5G). Furthermore, the dedifferentiation TFs, including MITF and LEF1, were downregulated early after two days of BRAFi treatment, whereas SOX10 and MEF2C were reduced upon the development of acquired resistance (SDR), which was also when the differentiation score was greatly reduced. This suggested that SOX10 and MEF2C downregulation are required or are the main contributors to the dedifferentiation gene expression signature. Overall, these data support the notion that PD-L1 $1_{\mathrm{CON}}$ expression occurs at the proliferative stage of drug resistance and that this is mediated by a transcriptome reprogramming, and is accompanied by increases in TNF and IFN signalling along with the IRF1 expression. Moreover, these expression changes further increase when resistance is stabilised, at which point the differentiation expression signature is downregulated. 
A

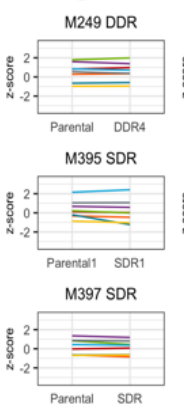
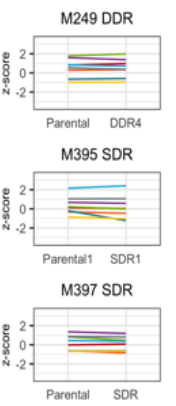$$
\text { Cell Line: BRAF mutation resistance }
$$

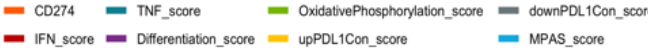

C

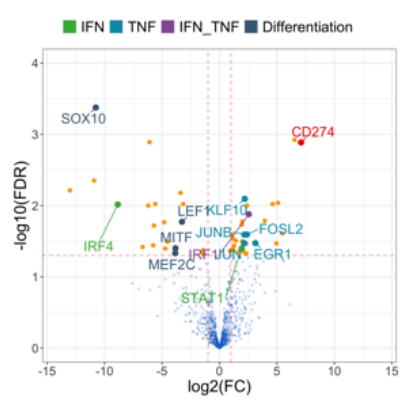

B Cell Line:transcriptome reprogrammed resistance

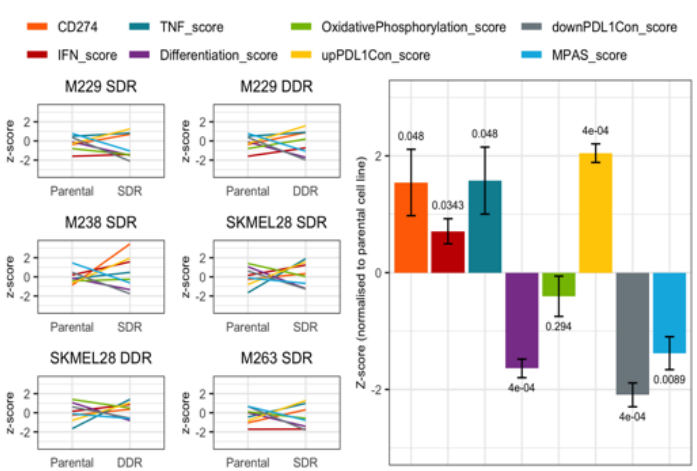

E

D Cell Line: BRAF mutation resistance

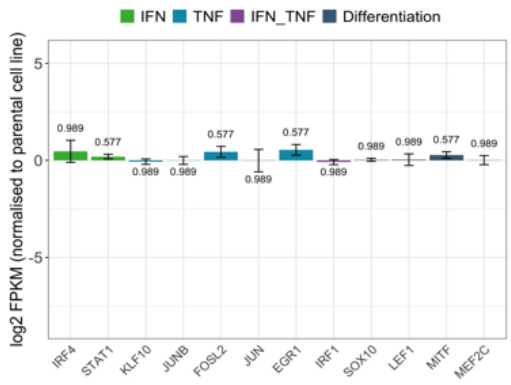

Cell Line:transcriptome reprogrammed resistance

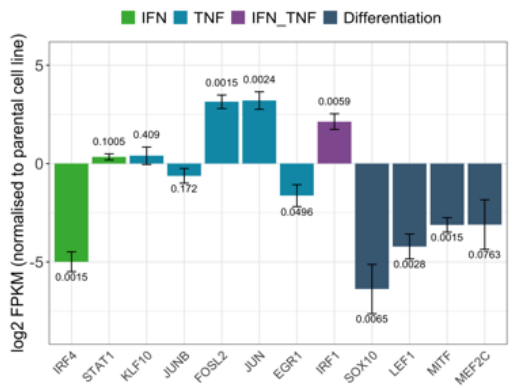

F

- OxidativePhosphorylation_score downPDL1Con_score

M229

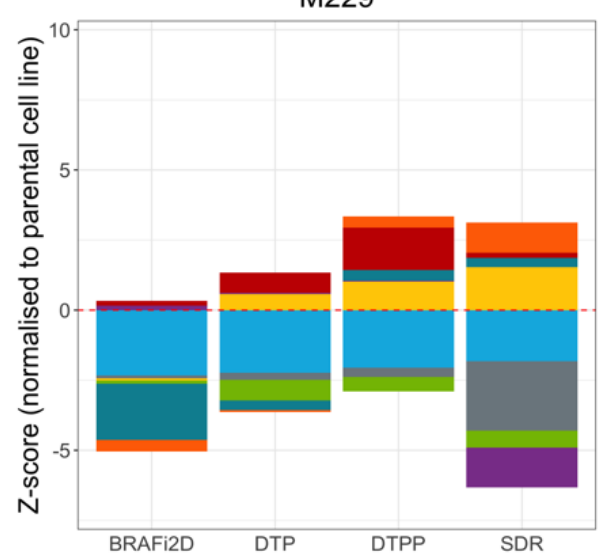

G

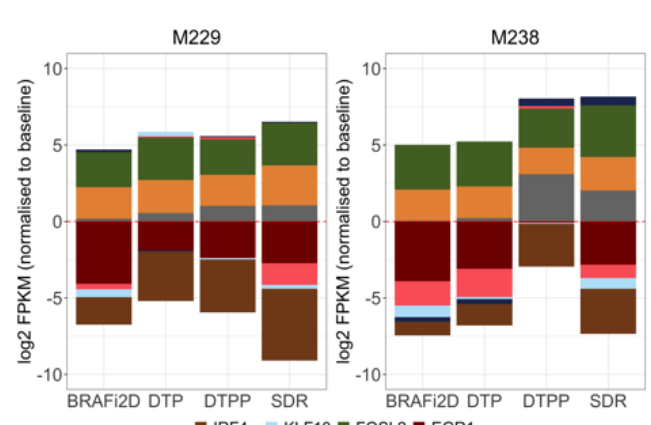

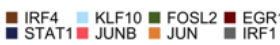

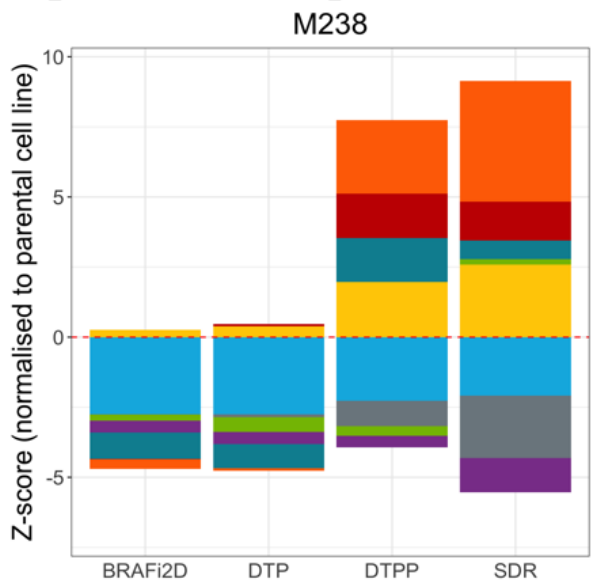

$\mathrm{H}$

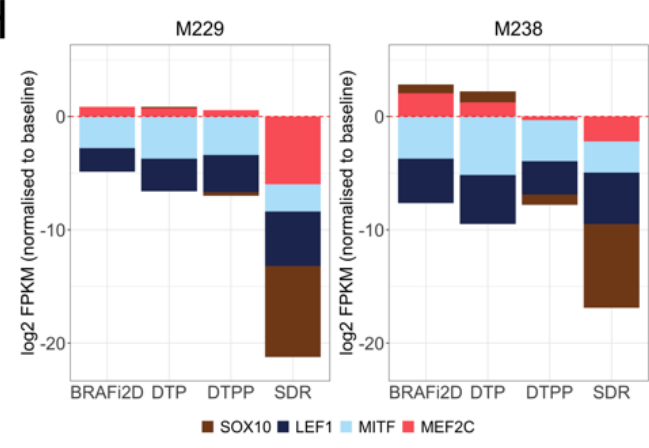

Figure 5. PD-L1 $\mathrm{CON}$ expression is associated with treatment resistance to MAPK inhibitors when associated with transcriptome reprogramming. (A,B) Five melanoma cell lines that acquired BRAF mutations (splicing and copy number amplification) 
upon development of resistance and six melanoma cell lines that did not acquire mutations but exhibited a transcriptome reprogrammed state upon development of resistance are shown. CD274 expression and seven scores which includes the IFN score, TNF score, differentiation score, oxidative phosphorylation score, the MPAS score, and the up and down PD-L1 ${ }_{C O N}$ scores are shown for the parental (before treatment) and after BRAFi resistance (SDR $=$ single drug resistance, $D D R=$ double drug resistance). The bar plots show values normalised to the corresponding baseline sample. Bar plot values are averages across all samples within either the BRAF mutation resistance group or the transcriptome reprogrammed resistance group. FDR adjusted $p$ values for paired $t$ test are shown for each bar. Error bars represent standard error. (C) Volcano plot shows the TFs that were differentially expressed in the PD-L1 $1_{\mathrm{CON}}$ samples and in addition, involved in IFN signalling, TNF signalling, both IFN and TNF signalling (labelled as "IFN_TNF"), and the melanocyte differentiation pathway. The CD274 gene is shown in red. (D,E) Bar plots shows the expression changes of TFs (involved in the IFN, TNF signalling, and melanocyte differentiation) following acquired resistance with either BRAF mutations or transcriptome reprogramming in melanoma cell lines. Error bars represents standard error. (F) Cumulative barplot shows the CD274 expression and the seven scores at different stages of BRAFi resistance for the M229 and M238 melanoma cell lines. Values are normalised to the parental cell line, and resistance stages include (1) two days of BRAFi treatment (BRAFi2D), (2) Drug Tolerant Persisters (DTP) where a small subpopulation of persisting cells remain, (3) Drug Tolerant Proliferative Persisters (DTPP) where proliferation was regained, and (4) single drug resistance (SDR), a permanent resistant state to BRAFi. (G,H) Cumulative barplot shows the mRNA expression changes of TFs involved in the IFN signalling, TNF signalling, and melanocyte differentiation at different stages of BRAFi resistance for the M229 and M238 melanoma cell lines.

\subsection{The PD-L1 CON Expression Signature Is Associated with Transcriptomic Reprogramming of Melanomas following MAPK Inhibitor Resistance in Patients}

We next investigated whether the PD-L1 $\mathrm{CON}$ expression signature occurs in melanomas from patients who develop acquired resistance to MAPK inhibitor treatment. To address this, we analysed RNA-Seq data from matched pre- and post-treatment tumour biopsies, following progression on treatment [19]. We compared melanomas with BRAF mutational resistance mechanisms (BRAF splicing and amplification) to melanomas lacking known mutations that drive resistance. In melanomas where no mutational resistance mechanism was identified, we observed the same direction of up and down gene expression changes to the PD-L1 CON cell lines, and to the transcriptomic changes of Song and colleagues' dataset, where resistance was mediated by transcriptomic reprogramming. This consisted of elevated levels of CD274 expression, and elevated IFN, TNF, and the "up" PD-L1 CON scores (normalised to matched pre-treatment tumours from the same patient), while oxidative phosphorylation, down PD-L1 $1_{\mathrm{CON}}$, and differentiation scores were reduced (Figure S7A). In addition, there were lower levels of the differentiation related TFs and higher levels of expression of JUN, IRF1, JUNB, and FOSL2 observed in melanomas with no BRAF mutational resistance mechanisms (normalised to patient matched pre-treatment tumours), compared to melanomas containing BRAF mutational resistance mechanisms (Figure S7B). These results support the notion that the PD-L1 ${ }_{C O N}$ expression signature accompanies transcriptomic reprogramming and treatment resistance in melanomas lacking a $B R A F$ mutational resistance mechanism.

\section{Discussion}

The use of targeted therapies based on MAPK pathway inhibition, or of anti-PD1 immunotherapy, has improved melanoma patient survival, although drug resistance greatly limits long-term survival for most patients with advanced melanoma. A large number of mutational and non-mutational (transcriptomic or epigenetic) resistance mechanisms have been identified in melanoma $[5,19,51,52]$. Moreover, these have been associated with a cell-intrinsic upregulation of the immunosuppressive PD-L1 protein $[4,5]$. In the present study, to explore how PD-L1 expression is associated with transcriptomic reprogramming and drug resistance, we have assessed the transcriptomic landscape of melanoma cell lines with constitutively high levels of PD-L1 expression (PD-L1 $1_{C O N}$ ). Overall, our study has shown that PD-L1 $1_{C O N}$ cells have many similarities to melanoma cell lines and patient tumours that exhibit intrinsically upregulated PD-L1 expression following the development of resistance to MAPK pathway inhibitors via non-mutational and/or epigenetic 
mechanisms. Our research reveals that this similarity is inclusive of a gene expression profile corresponding to dedifferentiation, and active cytokine (TNF and IFN) signalling pathways. Dedifferentiation is a non-mutational mechanism of drug resistance, which melanomas commonly exploit. There did not appear to be an association of transcriptomic reprogramming with the patient's sex, or with the tumour stage, although there were a limited number of samples in our analysis. Consistent with other studies showing that treatment of melanoma and melanocytes cells with TNF- $\alpha$ and IFN- $\gamma$ cytokines can induce a dedifferentiated state, we have found that the dedifferentiated state of PD-L1 $1_{\mathrm{CON}}$ cells is associated with constitutively active IFN and TNF signalling pathways $[17,37,46,53]$. Furthermore, given that the PD-L1 $1_{\mathrm{CON}}$ and $\mathrm{PD}-\mathrm{L} 1_{\mathrm{IND}}$ cell line groups used in this study were classified into these groups independent of $B R A F$ or NRAS mutation status (four cell lines were $B R A F$ mutant, two NRAS mutant cell lines, and one BRAF/NRAS wild-type), these data suggest that the presence of the BRAF or NRAS genetic mutations did not influence the occurrence of a PD-L1 $1_{\mathrm{CON}}$ transcriptomic expression pattern, and lend support to the notion that transcriptional or epigenetic factors can over-ride mutation-driven resistance in the absence of a targeted drug treatment.

It is important to note that almost all of the PD-L1 $1_{\mathrm{CON}}$ melanoma cell lines (six out of seven) in this study had not been previously exposed to targeted inhibitors either in the patient (in vivo) or in vitro. Nevertheless, each PD-L1 $1_{\mathrm{CON}}$ cell line contained the "resistant" gene expression signature. This suggests that stress factors, other than targeted inhibitor drugs, may have induced the resistant transcriptional profile. This could include stresses, such as hypoxia, or treatment using chemotherapeutic drugs in the patients before the cell lines were initiated. The clinical relevance of these findings is that, in some patients, PDL1 $\mathrm{CON}$ cells may occupy a subpopulation of cells in the tumour prior to targeted inhibitor therapy, which could then result in innate resistance. Moreover, one of the PD-L1 ${ }_{\mathrm{CON}}$ melanoma cell lines, which was previously exposed to MAPK pathway inhibitors (i.e., CM143-post), had a related cell line from the same patient, which also exhibited PD-L1 CON expression prior to treatment exposure (CM143-pre), suggesting that, in this patient, a PDL1 $1_{\mathrm{CON}}$ transcriptomic pattern may have represented a pre-existing subpopulation of cells that was present prior to treatment, and which gave rise to the drug-resistant phenotype.

\section{Conclusions}

Our study offers new insights into transcriptomic patterns associated with PD-L1 $1_{\mathrm{CON}}$ melanoma cell lines, the expression of relevant TFs, and reprogramming of melanoma cells towards a drug resistant phenotype. For instance, SOX10 and MITF are master TF regulators of melanocyte differentiation, whereby their downregulation can promote dedifferentiation and confer resistance to MAPK pathway inhibitors. This then results in activation of oncogenic signalling pathways, providing alternatives to the MAPK pathway, such as pathways driven by RTKs [16,52,54-57]. Both SOX10 and MITF were significantly downregulated in the PD-L1 $1_{\mathrm{CON}}$ cells, along with a dedifferentiation gene expression profile and increased expression of RTK genes which highlights the importance of PD$\mathrm{L}_{\mathrm{CON}}$ expression during adaptive drug resistance. Moreover, TFs that were significantly upregulated consisted of AP-1 (JUN and FOSL2), which have recently been shown to not only mediate the TNF signalling pathways, but also to alter the transcriptional and enhancer chromatin landscape by serving as pioneer TFs [58-60]. Transcriptomic reprogramming and differentiation often involves pioneer TFs, which facilitate and alter access to distinct regulatory elements, and consequently alter the chromatin landscape [61]. Moreover, SOX9 has also been shown to be a pioneer TF that mediates stem cell plasticity [62-64], and we found this was significantly upregulated in the PD-L1 $1_{\mathrm{CON}}$ cells. Finally, this study also provides foundations for further research to investigate mechanistic pathways (such as chromatin remodeling) leading to transcriptional reprogramming and the involvement of gene expression signatures, such as $\mathrm{PD}-\mathrm{L} 1_{\mathrm{CON}}$, in cancer drug-resistant phenotypes. 
Supplementary Materials: The following are available online at https:/ / www.mdpi.com/article/ 10.3390/cancers13174250/s1, Table S1: Information on the mutation status and the PD-L1 IND and PD-L1 $1_{\mathrm{CON}}$ group, Table S2: The PD-L1 $1_{\mathrm{CON}}$ signature genes for all protein-coding genes and lncRNAs, Figure S1: (A,B) PD-L1 protein and mRNA expression in the PD-L1 IND samples $(n=10)$ in comparison to the PD-L1 $1_{\mathrm{CON}}(n=7)$ melanoma cell lines. The bars represent Median Fluorescence Intensity (MFI) for PD-L1 staining normalised to isotype control by subtraction followed by $\log _{2}$ transformation: $\log _{2}\left(\mathrm{MFI}_{\text {antibody }}-\mathrm{MFI}_{\text {isotype }}\right)$. The mRNA expression levels are $\log _{2}$ transformed TMM normalized values. (C) Correlation between the PD-L1 protein and mRNA expression. (D) PD-L1 protein levels prior to (control group) and following one day of IFN $\gamma$ treatment for six out of ten PD-L1 $1_{\text {IND samples. }}$ In four out of the ten cell lines PDL1 had previously shown to be inducible with IFNg [6]. The paired $\mathrm{t}$ test $p$ value is shown, Figure S2: $(\mathrm{A}, \mathrm{B})$ Volcano plot showing the differentially expressed protein-coding genes (A) and lncRNAs (B). The top 10 differentially expressed gene names are shown and moreover the CD274 (PD-L1) and PDCD1LG2 genes (PD-L2) are shown. The x-axis represents $\log _{2}$ fold change and the $y$-axis represents the $-\log _{10}$ FDR adjusted $p$ value. (C) The CD274 $\left(\log _{2}\right.$ fold change $=7.1$, FDR adjusted $p$ value $=0.001)$ and $(D)$ PDCD1LG2 mRNA expression $\left(\log _{2}\right.$ fold change $=5.0$, FDR adjusted $p$ value $=0.03)$ in the PD-L1 ${ }_{\mathrm{CON}}$ in comparison to the PD-L1 $1_{\mathrm{IND}}$ melanoma cell lines, Figure S3: (A) Barcode plots showing eight gene sets with differential expression changes in the PD-L1 $1_{C O N}$ melanoma cell lines (red or right side) compared to the PD-L1 $1_{\text {IND }}$ cell lines (blue or left side). The $x$-axis shows the ranked list of all protein-coding genes according to $\log _{2}$ fold-change. The vertical bars on the $x$-axis represent the genes that are included in the genesets. The y-axis shows the enrichment score. The FDR adjusted $p$ values using the CAMERA test are shown. (B) Correlation of $C D 274$ expression with the oxidation phosphorylation score in the four external melanoma cell line datasets. The samples (x-axis) are ranked according to CD274 expression ( $\log _{2}$ transformed and corresponds to the right side y-axis) and the CD274 expression level is shown by the black line. Grey vertical bars indicate the oxidative phosphorylation scores and corresponds to the left side y-axis. The moving average of the oxidative phosphorylation score is represented by the blue line. The Pearson correlation between the moving average and CD274 expression is shown. (C) The x-axis shows the significantly upregulated $(n=462)$, downregulated $(n=298)$ protein-coding genes and the upregulated $(n=106)$ and downregulated $(n=71)$ lncRNAs in the PD-L1 $\mathrm{CON}$ samples compared to the PD-L1 IND samples. The y-axis shows the correlation (Pearson) of the differentially expressed genes with the oxidative phosphorylation score. The $\mathrm{x}$-axis is ranked according the lowest to highest correlation. The percentage value of genes higher than 0.25 or lower than -0.25 Pearson correlation value out of all genes in the respective gene set are shown, Figure S4: Hierarchical clustering of the PD-L1 $1_{\mathrm{CON}}$ and PD-L1 $1_{\text {IND }}$ melanoma cell lines using melanoma invasive expression signature genes. The gene signatures were derived from two independent studies: (A) Widmer et al. [45] and (B) Jeff et al. [44], Figure S5: (A,B) Z-scores normalised to baseline (before stimulation) for CD274 expression, and the six scores related to PD-L1 $1_{\mathrm{CON}}$ expression are shown for eight melanoma cell lines following IFN- $\gamma$ stimulation for 2-5 weeks or TNF stimulation for 3 days (GSE152755). Four samples with a differentiated (pink dots) and four with a dedifferentiated (blue dots) expression signature at baseline are shown, Figure S6: (A) Hierarchical clustering of the PD-L1 $\mathrm{CON}$ and the PDL1 $1_{\text {IND }}$ samples using receptor tyrosine kinase genes. Receptor tyrosine kinase genes that were differentially expressed in the PD-L1 $1_{\mathrm{CON}}$ cell lines with an FDR adjusted $p$ value $<0.05$ are indicated by * whereas between 0.05 and 0.1 are indicated by + (B-I) mRNA expression levels of in the PD$\mathrm{L}_{\mathrm{CON}}$ samples compared to the PD-L1 $1_{\text {IND }}$ samples of various genes that have been found to confer resistance to BRAFi in BRAF mutant melanomas. FDR adjusted $p$ values are shown, Figure S7: (A) Z-scores normalised to baseline (pre-treatment tumour) for CD274 expression and the seven scores related to PD-L1 $\mathrm{CON}$ expression in the tumours that gained BRAF mutations upon acquired resistance (labelled "BRAF_mutation", green) in comparison to tumours that did not gain any known mutational resistance (labelled "No_mutation", purple). (B) Expression levels of 12 TFs in the tumours that gained BRAF mutations upon acquired resistance (labelled "BRAF_mutation") in comparison to tumours that did not gain any known mutational resistance (labelled "No_mutation"). All values were normalised to the baseline (pre-treatment) tumour from the same patient. The t-test $p$ value is shown. 
Author Contributions: Conceptualization, A.A., A.C., E.J.R., P.H. and M.R.E.; methodology, A.A.; software, G.G. and P.A.S.; formal analysis, A.A., J.M.; resources, P.H. and M.R.E.; data curation, A.A., J.M., G.G. and M.P.; writing-original draft preparation, A.A.; writing-review and editing, A.A., E.J.R., J.M., G.G., P.A.S., M.P., P.H., A.C. and M.R.E.; funding acquisition, A.C. and M.R.E. All authors have read and agreed to the published version of the manuscript.

Funding: This research was funded by grants from the Royal Society of New Zealand Rutherford Discovery Fellowship Program (to AC), the Maurice Wilkins Centre for Molecular Biodiscovery, the Health Research Council of New Zealand grant number 18-144, the Cancer Society of New Zealand grant number 16.06, and Cancer Research Trust NZ.

Institutional Review Board Statement: Ethical review and approval were waived for this study because the study used human cell lines, which had already been established, and human data from online data repositories, and all ethical approvals for use in this context had already been obtained.

Informed Consent Statement: Patient consent was waived due to the use of established human cell lines, and/or use of data from online data repositories.

Data Availability Statement: Transcriptomic data for PD-L1 $1_{\mathrm{CON}}$ and PDL1 $1_{\text {IND }}$ cell lines are available at Database: NCBI GEO, accession number GSE107622 and GSE153595.

Acknowledgments: The authors thank Sarah Diermeier and Nicole Cloonan for comments and suggestions, and Glen Boyle for providing the MM127, MM595 and COLOF239F melanoma cell lines.

Conflicts of Interest: The authors declare no conflict of interest. The funders had no role in the design of the study; in the collection, analyses, or interpretation of data; in the writing of the manuscript, or in the decision to publish the results.

\section{References}

1. Domingues, B.; Lopes, J.M.; Soares, P.; Populo, H. Melanoma treatment in review. Immunotargets Ther. 2018, 7, 35-49. [CrossRef]

2. Sun, C.; Mezzadra, R.; Schumacher, T.N. Regulation and Function of the PD-L1 Checkpoint. Immunity 2018, 48, 434-452. [CrossRef]

3. Robert, C.; Schachter, J.; Long, G.V.; Arance, A.; Grob, J.J.; Mortier, L.; Daud, A.; Carlino, M.S.; McNeil, C.; Lotem, M.; et al. Pembrolizumab versus Ipilimumab in Advanced Melanoma. N. Engl. J. Med. 2015, 372, 2521-2532. [CrossRef]

4. Audrito, V.; Serra, S.; Stingi, A.; Orso, F.; Gaudino, F.; Bologna, C.; Neri, F.; Garaffo, G.; Nassini, R.; Baroni, G.; et al. PDL1 up-regulation in melanoma increases disease aggressiveness and is mediated through miR-17-5p. Oncotarget 2017, 8, 15894-15911. [CrossRef]

5. Song, C.; Piva, M.; Sun, L.; Hong, A.; Moriceau, G.; Kong, X.; Zhang, H.; Lomeli, S.; Qian, J.; Yu, C.C.; et al. Recurrent Tumor Cell-Intrinsic and -Extrinsic Alterations during MAPKi-Induced Melanoma Regression and Early Adaptation. Cancer Discov. 2017, 7, 1248-1265. [CrossRef]

6. Chatterjee, A.; Rodger, E.J.; Ahn, A.; Stockwell, P.A.; Parry, M.; Motwani, J.; Gallagher, S.J.; Shklovskaya, E.; Tiffen, J.; Eccles, M.R.; et al. Marked Global DNA Hypomethylation Is Associated with Constitutive PD-L1 Expression in Melanoma. iScience 2018, 4, 312-325. [CrossRef] [PubMed]

7. Emran, A.A.; Chatterjee, A.; Rodger, E.J.; Tiffen, J.C.; Gallagher, S.J.; Eccles, M.R.; Hersey, P. Targeting DNA Methylation and EZH2 Activity to Overcome Melanoma Resistance to Immunotherapy. Trends Immunol. 2019, 40, 328-344. [CrossRef] [PubMed]

8. Lee, S.J.; Jang, B.C.; Lee, S.W.; Yang, Y.I.; Suh, S.I.; Park, Y.M.; Oh, S.; Shin, J.G.; Yao, S.; Chen, L.; et al. Interferon regulatory factor-1 is prerequisite to the constitutive expression and IFN-gamma-induced upregulation of B7-H1 (CD274). FEBS Lett. 2006, 580, 755-762. [CrossRef] [PubMed]

9. Garcia-Diaz, A.; Shin, D.S.; Moreno, B.H.; Saco, J.; Escuin-Ordinas, H.; Rodriguez, G.A.; Zaretsky, J.M.; Sun, L.; Hugo, W.; Wang, X.; et al. Interferon Receptor Signaling Pathways Regulating PD-L1 and PD-L2 Expression. Cell Rep. 2017, 19, 1189-1201. [CrossRef]

10. Spranger, S.; Spaapen, R.M.; Zha, Y.; Williams, J.; Meng, Y.; Ha, T.T.; Gajewski, T.F. Up-regulation of PD-L1, IDO, and T(regs) in the melanoma tumor microenvironment is driven by CD8(+) T cells. Sci. Transl. Med. 2013, 5, 200ra116. [CrossRef]

11. Donia, M.; Andersen, R.; Kjeldsen, J.W.; Fagone, P.; Munir, S.; Nicoletti, F.; Andersen, M.H.; Thor Straten, P.; Svane, I.M. Aberrant Expression of MHC Class II in Melanoma Attracts Inflammatory Tumor-Specific CD4+ T- Cells, Which Dampen CD8+ T-cell Antitumor Reactivity. Cancer Res. 2015, 75, 3747-3759. [CrossRef]

12. Shen, X.; Zhang, L.; Li, J.; Li, Y.; Wang, Y.; Xu, Z.X. Recent Findings in the Regulation of Programmed Death Ligand 1 Expression. Front. Immunol. 2019, 10, 1337. [CrossRef] [PubMed]

13. Chatterjee, A.; Stockwell, P.A.; Ahn, A.; Rodger, E.J.; Leichter, A.L.; Eccles, M.R. Genome-wide methylation sequencing of paired primary and metastatic cell lines identifies common DNA methylation changes and a role for EBF3 as a candidate epigenetic driver of melanoma metastasis. Oncotarget 2017, 8, 6085-6101. [CrossRef]

14. Johansson, P.; Pavey, S.; Hayward, N. Confirmation of a BRAF mutation-associated gene expression signature in melanoma. Pigment. Cell Res. 2007, 20, 216-221. [CrossRef] [PubMed] 
15. Hoek, K.S.; Schlegel, N.C.; Brafford, P.; Sucker, A.; Ugurel, S.; Kumar, R.; Weber, B.L.; Nathanson, K.L.; Phillips, D.J.; Herlyn, M.; et al. Metastatic potential of melanomas defined by specific gene expression profiles with no BRAF signature. Pigment. Cell Res. 2006, 19, 290-302. [CrossRef] [PubMed]

16. Muller, J.; Krijgsman, O.; Tsoi, J.; Robert, L.; Hugo, W.; Song, C.; Kong, X.; Possik, P.A.; Cornelissen-Steijger, P.D.; Geukes Foppen, M.H.; et al. Low MITF/AXL ratio predicts early resistance to multiple targeted drugs in melanoma. Nat. Commun. 2014, 5, 5712. [CrossRef]

17. Tsoi, J.; Robert, L.; Paraiso, K.; Galvan, C.; Sheu, K.M.; Lay, J.; Wong, D.J.L.; Atefi, M.; Shirazi, R.; Wang, X.; et al. Multi-stage Differentiation Defines Melanoma Subtypes with Differential Vulnerability to Drug-Induced Iron-Dependent Oxidative Stress. Cancer Cell. 2018, 33, 890-904.e5. [CrossRef] [PubMed]

18. Robinson, M.D.; McCarthy, D.J.; Smyth, G.K. edgeR: A Bioconductor package for differential expression analysis of digital gene expression data. Bioinformatics 2010, 26, 139-140. [CrossRef]

19. Hugo, W.; Shi, H.; Sun, L.; Piva, M.; Song, C.; Kong, X.; Moriceau, G.; Hong, A.; Dahlman, K.B.; Johnson, D.B.; et al. Non-genomic and Immune Evolution of Melanoma Acquiring MAPKi Resistance. Cell 2015, 162, 1271-1285. [CrossRef]

20. Kim, Y.J.; Sheu, K.M.; Tsoi, J.; Abril-Rodriguez, G.; Medina, E.; Grasso, C.S.; Torrejon, D.Y.; Champhekar, A.S.; Litchfield, K.; Swanton, C.; et al. Melanoma dedifferentiation induced by IFN-gamma epigenetic remodeling in response to anti-PD-1 therapy. J. Clin. Invest. 2021, 131. [CrossRef]

21. Colaprico, A.; Silva, T.C.; Olsen, C.; Garofano, L.; Cava, C.; Garolini, D.; Sabedot, T.S.; Malta, T.M.; Pagnotta, S.M.; Castiglioni, I.; et al. TCGAbiolinks: An R/Bioconductor package for integrative analysis of TCGA data. Nucleic Acids Res. 2016, 44, e71. [CrossRef] [PubMed]

22. Stockwell, P.A.; Chatterjee, A.; Rodger, E.J.; Morison, I.M. DMAP: Differential methylation analysis package for RRBS and WGBS data. Bioinformatics 2014, 30, 1814-1822. [CrossRef]

23. Chatterjee, A.; Stockwell, P.A.; Rodger, E.J.; Morison, I.M. Comparison of alignment software for genome-wide bisulphite sequence data. Nucleic Acids Res. 2012, 40, e79. [CrossRef] [PubMed]

24. Chatterjee, A.; Ahn, A.; Rodger, E.J.; Stockwell, P.A.; Eccles, M.R. A Guide for Designing and Analyzing RNA-Seq Data. Methods Mol. Biol. 2018, 1783, 35-80. [CrossRef] [PubMed]

25. Zheng, H.; Brennan, K.; Hernaez, M.; Gevaert, O. Benchmark of long non-coding RNA quantification for RNA sequencing of cancer samples. Gigascience 2019, 8. [CrossRef]

26. Bray, N.L.; Pimentel, H.; Melsted, P.; Pachter, L. Near-optimal probabilistic RNA-seq quantification. Nat. Biotechnol. 2016, 34, 525-527. [CrossRef] [PubMed]

27. Soneson, C.; Love, M.I.; Robinson, M.D. Differential analyses for RNA-seq: Transcript-level estimates improve gene-level inferences. F1000Research 2015, 4, 1521. [CrossRef]

28. Lun, A.T.; Chen, Y.; Smyth, G.K. It's DE-licious: A Recipe for Differential Expression Analyses of RNA-seq Experiments Using Quasi-Likelihood Methods in edgeR. Methods Mol. Biol. 2016, 1418, 391-416. [CrossRef]

29. Liberzon, A.; Birger, C.; Thorvaldsdottir, H.; Ghandi, M.; Mesirov, J.P.; Tamayo, P. The Molecular Signatures Database (MSigDB) hallmark gene set collection. Cell Syst. 2015, 1, 417-425. [CrossRef]

30. Wu, D.; Smyth, G.K. Camera: A competitive gene set test accounting for inter-gene correlation. Nucleic Acids Res. 2012, 40, e133. [CrossRef]

31. Barbie, D.A.; Tamayo, P.; Boehm, J.S.; Kim, S.Y.; Moody, S.E.; Dunn, I.F.; Schinzel, A.C.; Sandy, P.; Meylan, E.; Scholl, C.; et al. Systematic RNA interference reveals that oncogenic KRAS-driven cancers require TBK1. Nature 2009, 462, 108-112. [CrossRef]

32. Hanzelmann, S.; Castelo, R.; Guinney, J. GSVA: Gene set variation analysis for microarray and RNA-seq data. BMC Bioinform. 2013, 14, 7. [CrossRef] [PubMed]

33. Chiappinelli, K.B.; Strissel, P.L.; Desrichard, A.; Li, H.; Henke, C.; Akman, B.; Hein, A.; Rote, N.S.; Cope, L.M.; Snyder, A.; et al. Inhibiting DNA Methylation Causes an Interferon Response in Cancer via dsRNA Including Endogenous Retroviruses. Cell 2015, 162, 974-986. [CrossRef] [PubMed]

34. Roulois, D.; Loo Yau, H.; Singhania, R.; Wang, Y.; Danesh, A.; Shen, S.Y.; Han, H.; Liang, G.; Jones, P.A.; Pugh, T.J.; et al. DNA-Demethylating Agents Target Colorectal Cancer Cells by Inducing Viral Mimicry by Endogenous Transcripts. Cell 2015, 162, 961-973. [CrossRef]

35. Rooney, M.S.; Shukla, S.A.; Wu, C.J.; Getz, G.; Hacohen, N. Molecular and genetic properties of tumors associated with local immune cytolytic activity. Cell 2015, 160, 48-61. [CrossRef] [PubMed]

36. Becht, E.; Giraldo, N.A.; Lacroix, L.; Buttard, B.; Elarouci, N.; Petitprez, F.; Selves, J.; Laurent-Puig, P.; Sautes-Fridman, C.; Fridman, W.H.; et al. Estimating the population abundance of tissue-infiltrating immune and stromal cell populations using gene expression. Genome Biol. 2016, 17, 218. [CrossRef]

37. Riesenberg, S.; Groetchen, A.; Siddaway, R.; Bald, T.; Reinhardt, J.; Smorra, D.; Kohlmeyer, J.; Renn, M.; Phung, B.; Aymans, P.; et al. MITF and c-Jun antagonism interconnects melanoma dedifferentiation with pro-inflammatory cytokine responsiveness and myeloid cell recruitment. Nat. Commun. 2015, 6, 8755. [CrossRef]

38. Gowrishankar, K.; Gunatilake, D.; Gallagher, S.J.; Tiffen, J.; Rizos, H.; Hersey, P. Inducible but not constitutive expression of PD-L1 in human melanoma cells is dependent on activation of NF-kappaB. PLoS ONE 2015, 10, e0123410. [CrossRef] 
39. Gao, J.; Shi, L.Z.; Zhao, H.; Chen, J.; Xiong, L.; He, Q.; Chen, T.; Roszik, J.; Bernatchez, C.; Woodman, S.E.; et al. Loss of IFN-gamma Pathway Genes in Tumor Cells as a Mechanism of Resistance to Anti-CTLA-4 Therapy. Cell 2016, 167, 397-404.e9. [CrossRef] [PubMed]

40. Zaretsky, J.M.; Garcia-Diaz, A.; Shin, D.S.; Escuin-Ordinas, H.; Hugo, W.; Hu-Lieskovan, S.; Torrejon, D.Y.; Abril-Rodriguez, G.; Sandoval, S.; Barthly, L.; et al. Mutations Associated with Acquired Resistance to PD-1 Blockade in Melanoma. N. Engl. J. Med. 2016, 375, 819-829. [CrossRef]

41. Alavi, S.; Stewart, A.J.; Kefford, R.F.; Lim, S.Y.; Shklovskaya, E.; Rizos, H. Interferon Signaling Is Frequently Downregulated in Melanoma. Front. Immunol. 2018, 9, 1414. [CrossRef]

42. Sucker, A.; Zhao, F.; Pieper, N.; Heeke, C.; Maltaner, R.; Stadtler, N.; Real, B.; Bielefeld, N.; Howe, S.; Weide, B.; et al. Acquired IFNgamma resistance impairs anti-tumor immunity and gives rise to T-cell-resistant melanoma lesions. Nat Commun. 2017, 8, 15440. [CrossRef]

43. Shin, D.S.; Zaretsky, J.M.; Escuin-Ordinas, H.; Garcia-Diaz, A.; Hu-Lieskovan, S.; Kalbasi, A.; Grasso, C.S.; Hugo, W.; Sandoval, S.; Torrejon, D.Y.; et al. Primary Resistance to PD-1 Blockade Mediated by JAK1/2 Mutations. Cancer Discov. 2017, 7, 188-201. [CrossRef]

44. Jeffs, A.R.; Glover, A.C.; Slobbe, L.J.; Wang, L.; He, S.; Hazlett, J.A.; Awasthi, A.; Woolley, A.G.; Marshall, E.S.; Joseph, W.R.; et al. A gene expression signature of invasive potential in metastatic melanoma cells. PLoS ONE 2009, 4, e8461. [CrossRef] [PubMed]

45. Widmer, D.S.; Cheng, P.F.; Eichhoff, O.M.; Belloni, B.C.; Zipser, M.C.; Schlegel, N.C.; Javelaud, D.; Mauviel, A.; Dummer, R.; Hoek, K.S. Systematic classification of melanoma cells by phenotype-specific gene expression mapping. Pigment. Cell Melanoma Res. 2012, 25, 343-353. [CrossRef]

46. Landsberg, J.; Kohlmeyer, J.; Renn, M.; Bald, T.; Rogava, M.; Cron, M.; Fatho, M.; Lennerz, V.; Wolfel, T.; Holzel, M.; et al. Melanomas resist T-cell therapy through inflammation-induced reversible dedifferentiation. Nature 2012, 490, $412-416$. [CrossRef] [PubMed]

47. Mehta, A.; Kim, Y.J.; Robert, L.; Tsoi, J.; Comin-Anduix, B.; Berent-Maoz, B.; Cochran, A.J.; Economou, J.S.; Tumeh, P.C.; Puig-Saus, C.; et al. Immunotherapy Resistance by Inflammation-Induced Dedifferentiation. Cancer Discov. $2018,8,935-943$. [CrossRef] [PubMed]

48. Lambert, S.A.; Jolma, A.; Campitelli, L.F.; Das, P.K.; Yin, Y.; Albu, M.; Chen, X.; Taipale, J.; Hughes, T.R.; Weirauch, M.T. The Human Transcription Factors. Cell 2018, 172, 650-665. [CrossRef]

49. Wang, Y.; Wang, H.; Yao, H.; Li, C.; Fang, J.Y.; Xu, J. Regulation of PD-L1: Emerging Routes for Targeting Tumor Immune Evasion. Front. Pharmacol. 2018, 9, 536. [CrossRef]

50. Zerdes, I.; Matikas, A.; Bergh, J.; Rassidakis, G.Z.; Foukakis, T. Genetic, transcriptional and post-translational regulation of the programmed death protein ligand 1 in cancer: Biology and clinical correlations. Oncogene 2018, 37, 4639-4661. [CrossRef]

51. Hugo, W.; Zaretsky, J.M.; Sun, L.; Song, C.; Moreno, B.H.; Hu-Lieskovan, S.; Berent-Maoz, B.; Pang, J.; Chmielowski, B.; Cherry, G.; et al. Genomic and Transcriptomic Features of Response to Anti-PD-1 Therapy in Metastatic Melanoma. Cell 2016, 165, 35-44. [CrossRef]

52. Shaffer, S.M.; Dunagin, M.C.; Torborg, S.R.; Torre, E.A.; Emert, B.; Krepler, C.; Beqiri, M.; Sproesser, K.; Brafford, P.A.; Xiao, M.; et al. Rare cell variability and drug-induced reprogramming as a mode of cancer drug resistance. Nature 2017, 546, 431-435. [CrossRef] [PubMed]

53. Natarajan, V.T.; Ganju, P.; Singh, A.; Vijayan, V.; Kirty, K.; Yadav, S.; Puntambekar, S.; Bajaj, S.; Dani, P.P.; Kar, H.K.; et al. IFN-gamma signaling maintains skin pigmentation homeostasis through regulation of melanosome maturation. Proc. Natl. Acad. Sci. USA 2014, 111, 2301-2306. [CrossRef] [PubMed]

54. Sun, C.; Wang, L.; Huang, S.; Heynen, G.J.; Prahallad, A.; Robert, C.; Haanen, J.; Blank, C.; Wesseling, J.; Willems, S.M.; et al. Reversible and adaptive resistance to BRAF(V600E) inhibition in melanoma. Nature 2014, 508, 118-122. [CrossRef] [PubMed]

55. Konieczkowski, D.J.; Johannessen, C.M.; Abudayyeh, O.; Kim, J.W.; Cooper, Z.A.; Piris, A.; Frederick, D.T.; Barzily-Rokni, M.; Straussman, R.; Haq, R.; et al. A melanoma cell state distinction influences sensitivity to MAPK pathway inhibitors. Cancer Discov. 2014, 4, 816-827. [CrossRef]

56. Verfaillie, A.; Imrichova, H.; Atak, Z.K.; Dewaele, M.; Rambow, F.; Hulselmans, G.; Christiaens, V.; Svetlichnyy, D.; Luciani, F.; Van den Mooter, L.; et al. Decoding the regulatory landscape of melanoma reveals TEADS as regulators of the invasive cell state. Nat. Commun. 2015, 6, 6683. [CrossRef]

57. Ji, Z.; Erin Chen, Y.; Kumar, R.; Taylor, M.; Jenny Njauw, C.N.; Miao, B.; Frederick, D.T.; Wargo, J.A.; Flaherty, K.T.; Jonsson, G.; et al. MITF Modulates Therapeutic Resistance through EGFR Signaling. J. Invest. Dermatol. 2015, 135, 1863-1872. [CrossRef]

58. Martinez-Zamudio, R.I.; Roux, P.F.; de Freitas, J.; Robinson, L.; Dore, G.; Sun, B.; Belenki, D.; Milanovic, M.; Herbig, U.; Schmitt, C.A.; et al. AP-1 imprints a reversible transcriptional programme of senescent cells. Nat. Cell Biol. 2020, 22, 842-855. [CrossRef]

59. Bi, M.; Zhang, Z.; Jiang, Y.Z.; Xue, P.; Wang, H.; Lai, Z.; Fu, X.; De Angelis, C.; Gong, Y.; Gao, Z.; et al. Enhancer reprogramming driven by high-order assemblies of transcription factors promotes phenotypic plasticity and breast cancer endocrine resistance. Nat. Cell Biol. 2020, 22, 701-715. [CrossRef]

60. Watt, A.C.; Cejas, P.; DeCristo, M.J.; Metzger-Filho, O.; Lam, E.Y.N.; Qiu, X.; BrinJones, H.; Kesten, N.; Coulson, R.; Font-Tello, A.; et al. CDK4/6 inhibition reprograms the breast cancer enhancer landscape by stimulating AP-1 transcriptional activity. Nat. Cancer 2020. [CrossRef] 
61. Choukrallah, M.A.; Matthias, P. The Interplay between Chromatin and Transcription Factor Networks during B Cell Development: Who Pulls the Trigger First? Front. Immunol. 2014, 5, 156. [CrossRef] [PubMed]

62. Sharma, A.; Cao, E.Y.; Kumar, V.; Zhang, X.; Leong, H.S.; Wong, A.M.L.; Ramakrishnan, N.; Hakimullah, M.; Teo, H.M.V.; Chong, F.T.; et al. Longitudinal single-cell RNA sequencing of patient-derived primary cells reveals drug-induced infidelity in stem cell hierarchy. Nat. Commun. 2018, 9, 4931. [CrossRef]

63. Adam, R.C.; Yang, H.; Rockowitz, S.; Larsen, S.B.; Nikolova, M.; Oristian, D.S.; Polak, L.; Kadaja, M.; Asare, A.; Zheng, D.; et al. Pioneer factors govern super-enhancer dynamics in stem cell plasticity and lineage choice. Nature 2015, 521, 366-370. [CrossRef] [PubMed]

64. Ge, Y.; Gomez, N.C.; Adam, R.C.; Nikolova, M.; Yang, H.; Verma, A.; Lu, C.P.; Polak, L.; Yuan, S.; Elemento, O.; et al. Stem Cell Lineage Infidelity Drives Wound Repair and Cancer. Cell 2017, 169, 636-650.e14. [CrossRef] [PubMed] 